\title{
Topological defects in nematics: fundamentals and applications
}

Samo Kralj ${ }^{1,2, *}$, Sasa Harkai², Luka Mesarec ${ }^{3}$, Aleš Iglič3 $^{3}$, Zdravko Kutnjak $^{2}$, Charles Rosenblatt ${ }^{4}$

${ }^{1}$ Faculty of Natural Sciences and Mathematics, University of Maribor, Koroška 160, 2000 Maribor, Slovenia

${ }^{2}$ Condensed Matter Physics, Jožef Stefan Institute, Jamova 39, 1000 Ljubljana, Slovenia ${ }^{3}$ Laboratory of Biophysics, Faculty of Electrical Engineering, University of Ljubljana, Tržaška 25, 1000 Ljubljana, Slovenia

${ }^{4}$ Department of Physics, Case Western Reserve University Cleveland, 44106 Ohio, USA

*Corresponding author: samo.kralj@um.si 


\section{Abstract:}

Topological defects (TDs) constitute topologically protected frustrated regions in a host field of an ordered manifold. They are ubiquitous in nature and appear at all scales, including the realms of particle physics, condensed matter, cosmology... We demonstrate that a simple plane parallel cell that confines a nematic liquid crystal (LC) could host diverse complex and multistable configurations of TDs, which we stabilized using the AFM scribing method. These competitive states could be reversibly and robustly reconfigured by appropriate external electric fields. Furthermore, we show that complex lattices of line defects, which are otherwise unstable or stable in a narrow interval of temperatures, could be stabilized efficiently by doping LCs with appropriate nanoparticles. We demonstrate that such TD configurations have potential for diverse applications, particularly in nanoand biotechnology: e.g., for nanotechnology-based devices based on reconfigurable conducting nanowires, tunable photonic devices, sensitive sensors... Furthermore, our study of TDs might provide some insight into still unresolved problems of fundamental physics. Namely, LCs could exhibit so-called "chargeless" twist disclinations, which commonly decay into a defectless state. Twist TDs could simultaneously act as defects and antidefects [3], and such neighboring pairs could be mutually annihilated. These configurations bear some resemblance to intriguing Majorana particles.

Keywords: topological defects; liquid crystals; nanoparticles 


\section{INTRODUCTION}
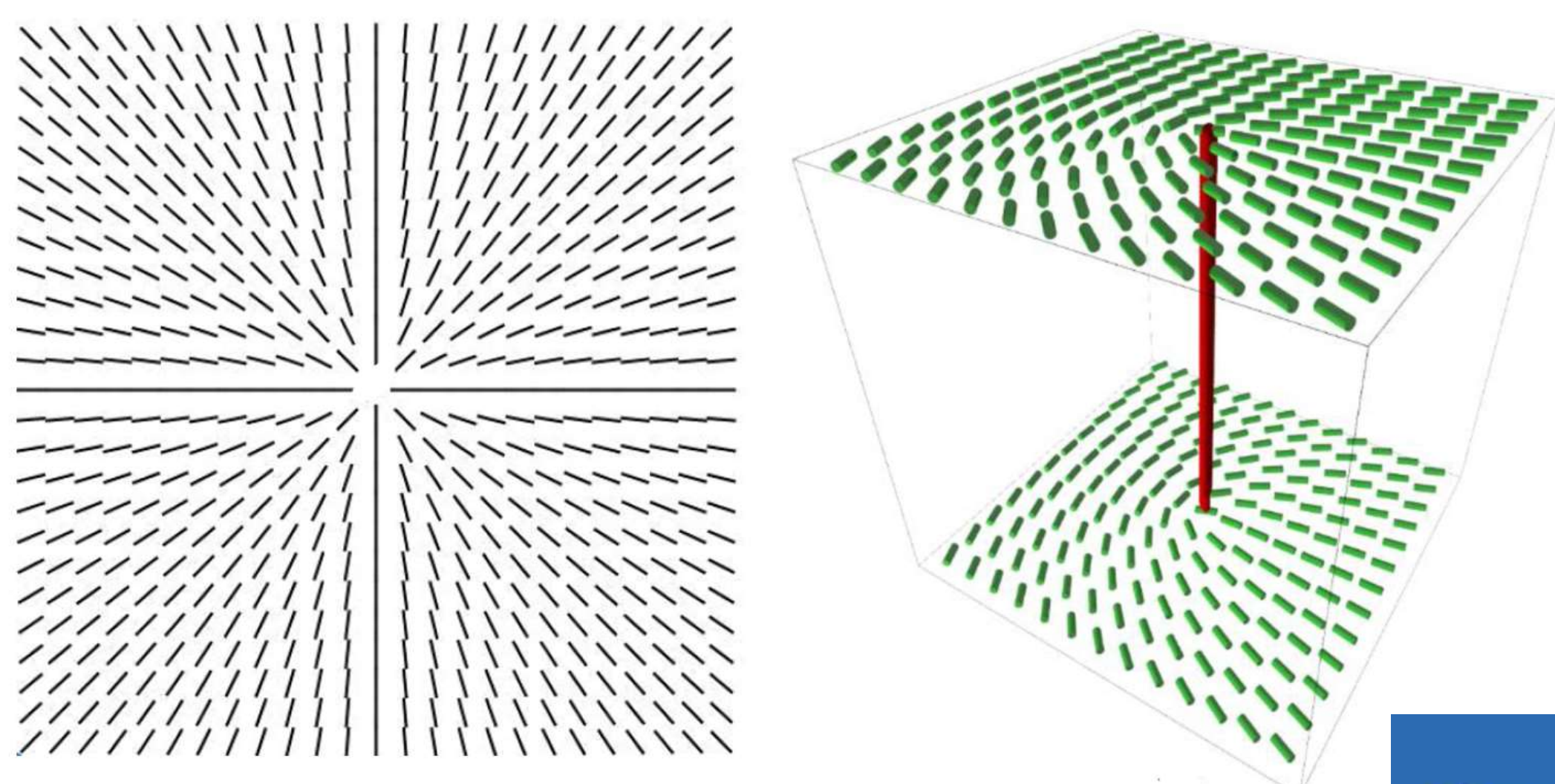

Crystals 2020 


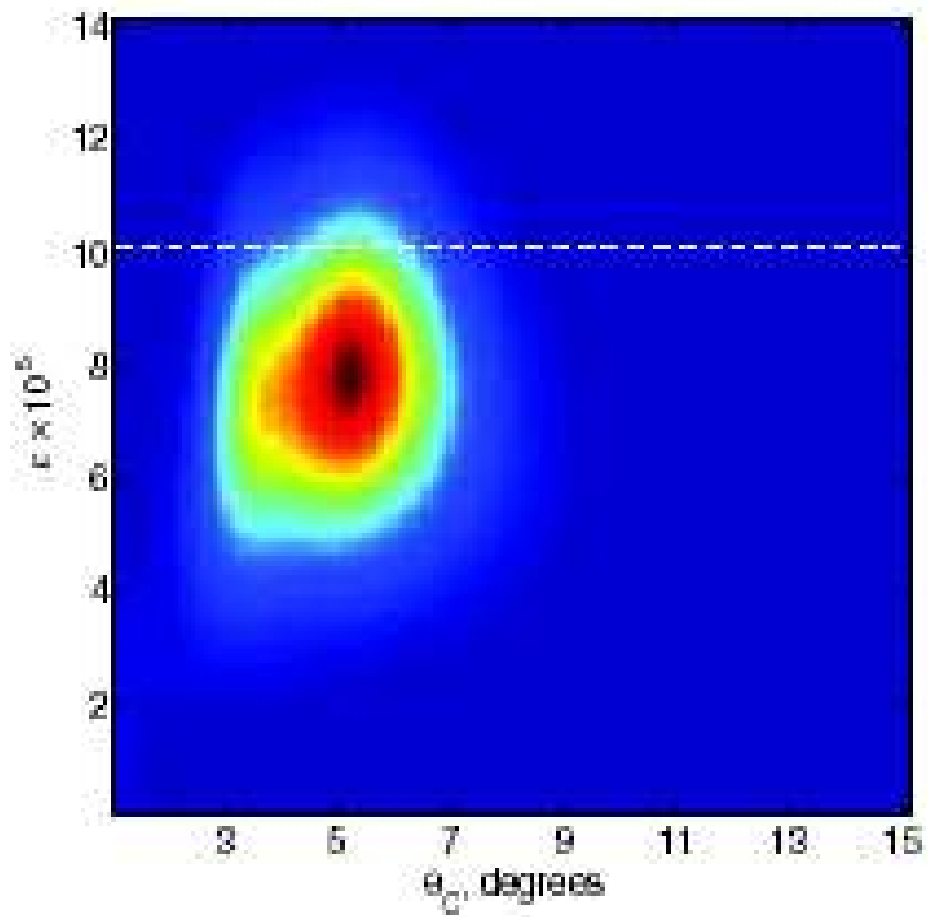

\section{A Cosmic Microwave Background Feature Consistent with a Cosmic Texture}

M. Cruz ${ }^{1, z_{*}}$ N. Turok, ${ }^{3}$ P. Vielva, ${ }^{1}$ E. Martínez-González, ${ }^{1}$ M. Hobson ${ }^{4}$

The Cosmic Microwave Background provides our most ancient image of the universe and our best tool for studying its early evolution. Theories of high-energy physics predict the formation of various types of topological defects in the very early universe, including cosmic texture, which would generate hot and cold spots in the Cosmic Microwave Background. We show through a Bayesian statistical analysis that the most prominent $5^{\circ}$-radius cold spot observed in all-sky images, which is otherwise hard to explain, is compatible with having being caused by a texture From this model, we constrain the fundamental symmetry-brea king energy scale to be $\phi_{0} \approx 8.7 \times 10^{15}$ gigaelectron volts. If confimed, this detection of a cosmic defect will probe physics at energies exceeding any conceivable terrestrial experiment. 


\title{
There are no particles, there are only fields
}

\author{
Art Hobson ${ }^{\text {a }}$ \\ Department of Physics, University of Arkansas, Fayetteville, AR, \\ ahobson@uark.edu
}

Quantum foundations are still unsettled, with mixed effects on science and society. By now it should be possible to obtain consensus on at least one issue: Are the fundamental constituents fields or particles? As this paper shows, experiment and theory imply unbounded fields, not bounded particles, are fundamental. This is especially clear for relativistic systems, implying it's also true of non-relativistic systems. Particles are epiphenomena arising from fields. Thus the Schroedinger field is a space-filling physical field whose value at any spatial point is the probability amplitude for an interaction to occur at that point.

$$
\text { Am.J.Phys. } 81 \text { (3), } 211 \text { (2013) }
$$


Typical Skyrmion

\section{Skyrmions : as $p, n$ in a pion-field}

\section{T. Skyrme,}

A unified field theory of mesons and bayrons, Nucl. Phys. 31, 556 (1962).

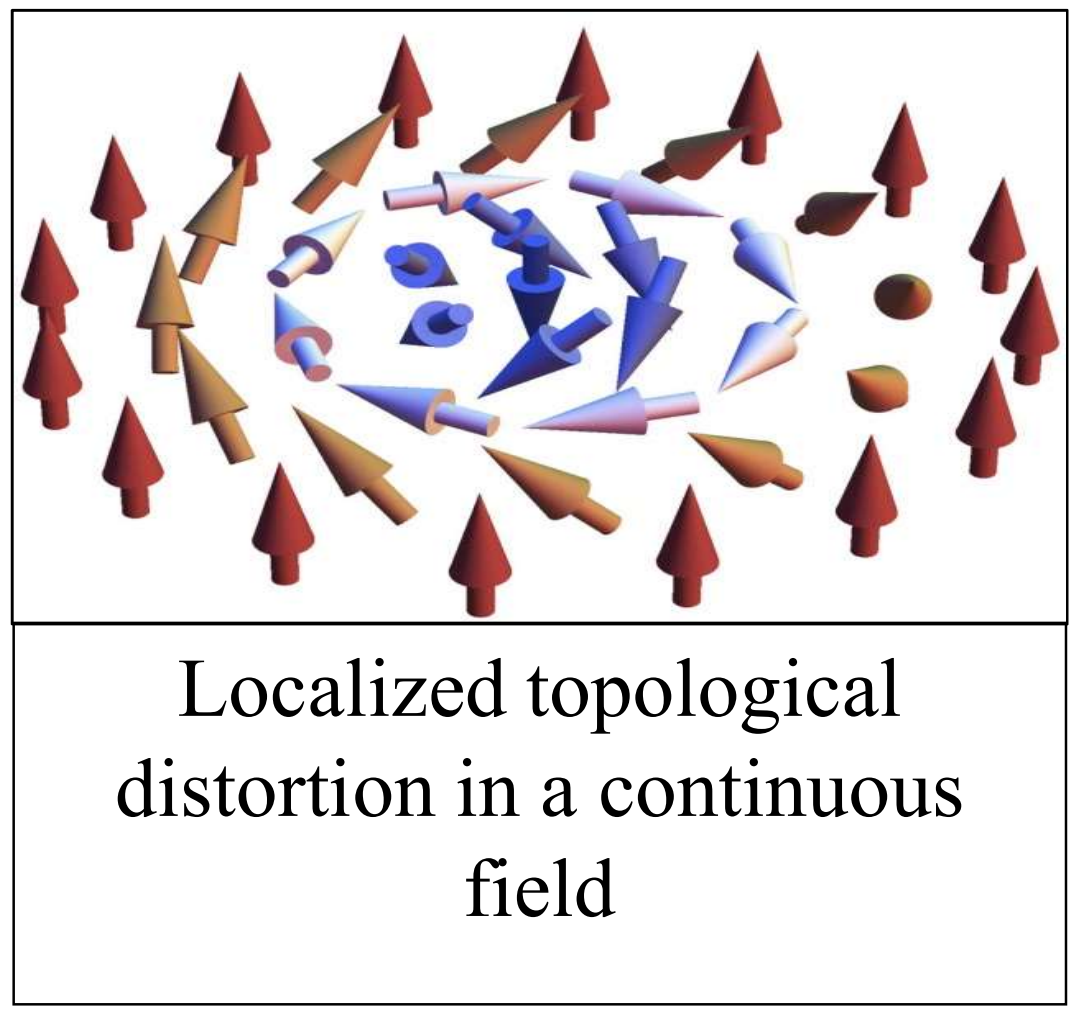

Crystals 2020 


\section{Applications}

\section{ARTICLES}

nature

https://doi.org/10.1038/541563-019-0512-3

materials

\section{Reconfiguration of three-dimensional liquid-} crystalline photonic crystals by electrostriction

Duan-Yi Guo ${ }^{1,4}$, Chun-Wei Chen ${ }^{2,4}$, Cheng-Chang Li', Hung-Chang Jau', Keng-Hsien Lin',

Ting-Mao Feng', Chun-Ta Wang1, Timothy J. Bunning ${ }^{3}$, lam Choon Khoo ${ }^{2 \star}{ }^{2 \star}$ and Tsung-Hsien Lin ${ }^{1 *}$
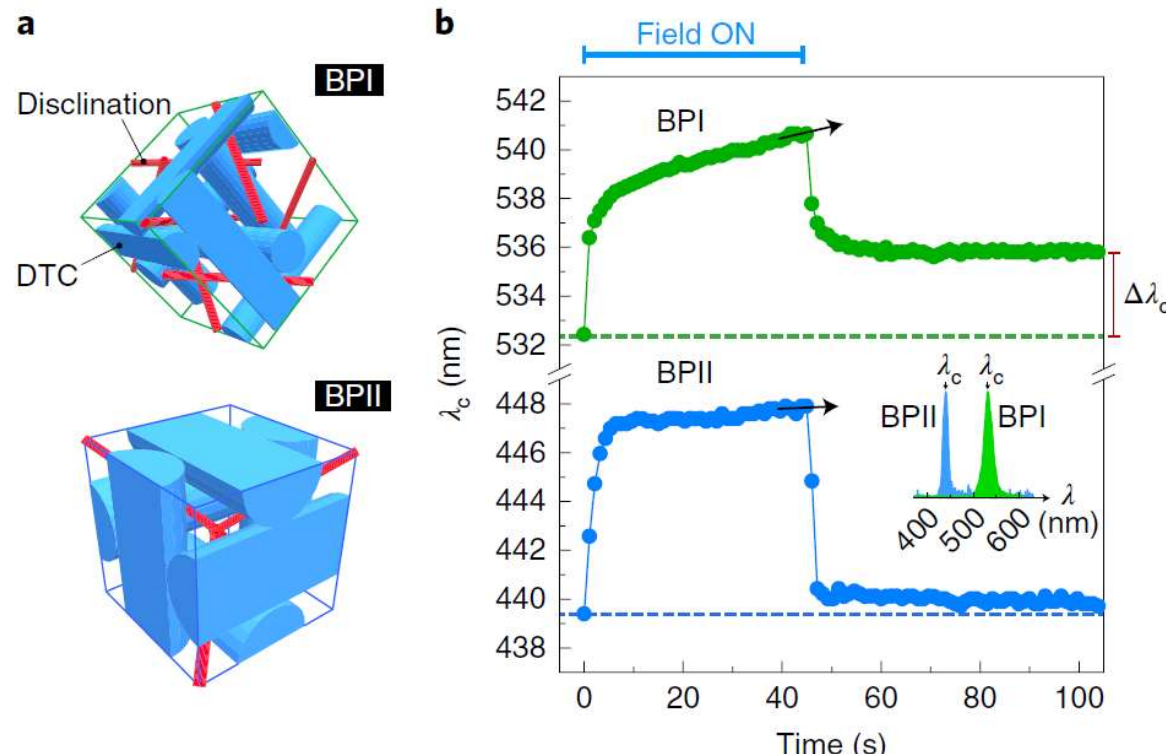
Adequate testbed - Liquid Crstals

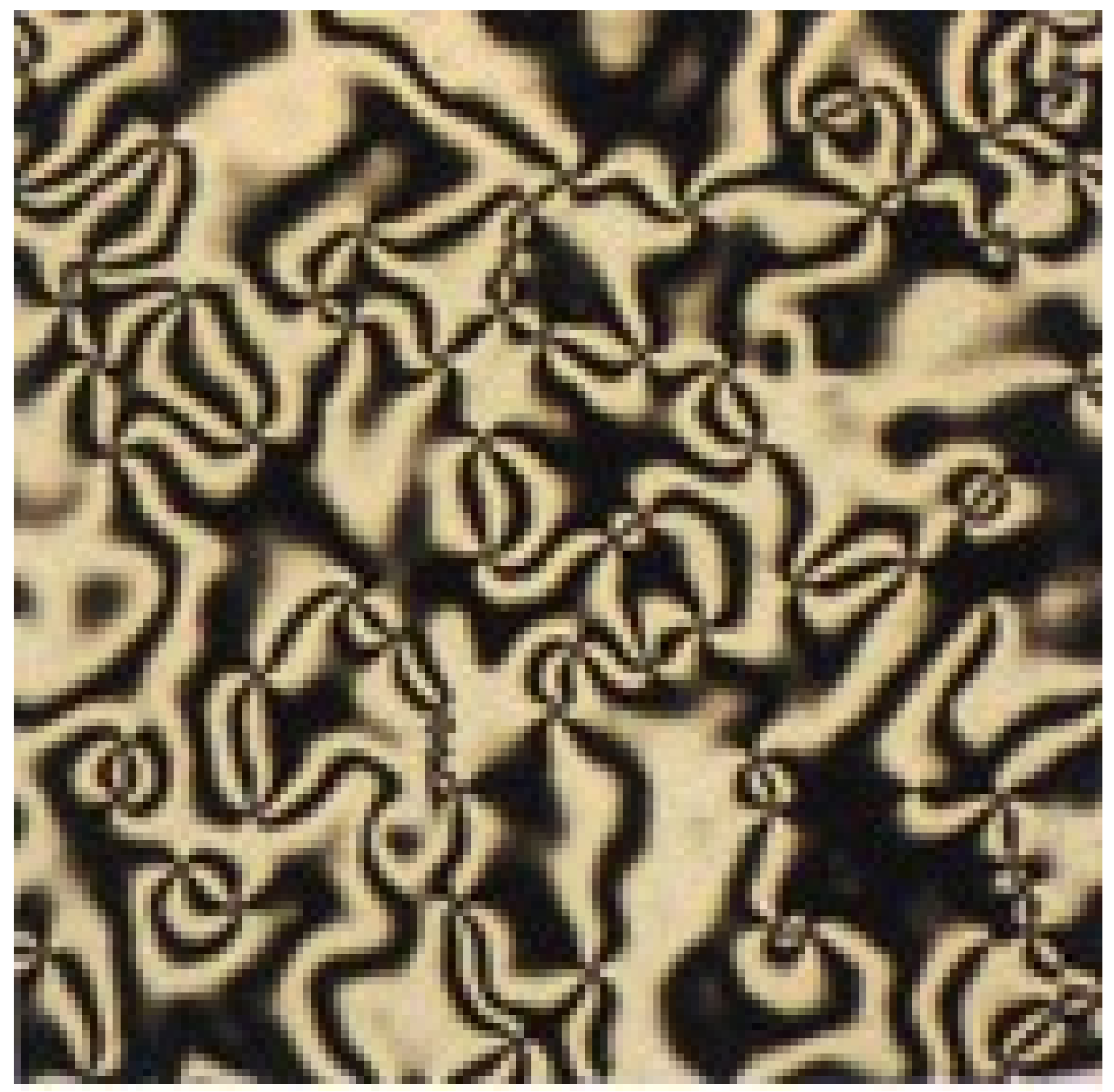

Crystals 


\section{CONTENT:}

Topological Defects in Liquid Crystals

- Order parameter : amplitude\&phase

- Topological charge

- Origin of topological defects

Stabilisation : confinement

Stabilisation : curvature

Intrinsic and extrinsic curvature

Stabilisation of "chargeless" topological defects

Stabilisation with nanoparticles

- Conclusions 


\section{Topological Defects in Liquid Crystals}
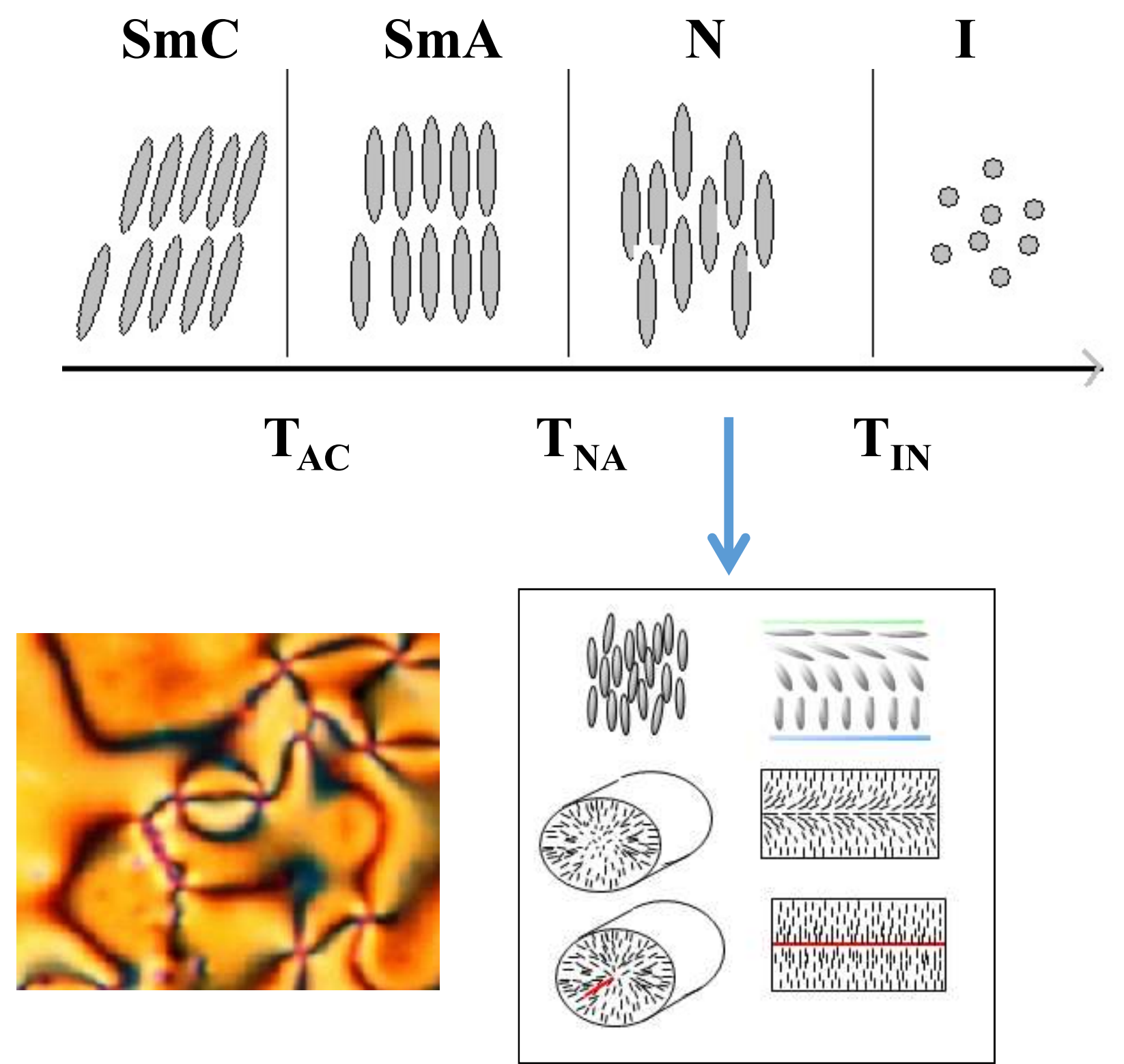

Crystals 


\section{Order parameter : amplitude\&phase}

Continuous symmetry breaking phase transition:

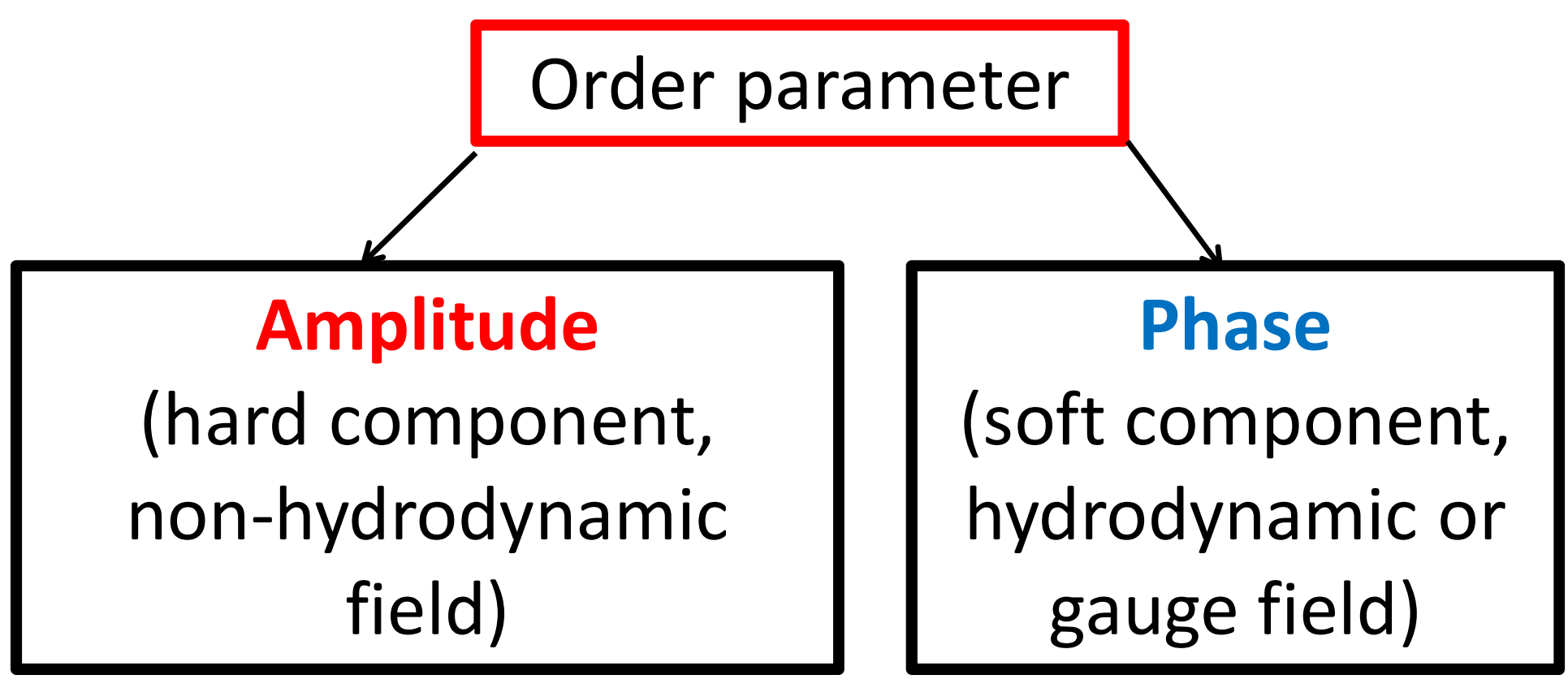

Palffy, Phys.Today 60, 54 (2007). 
Orientational order:

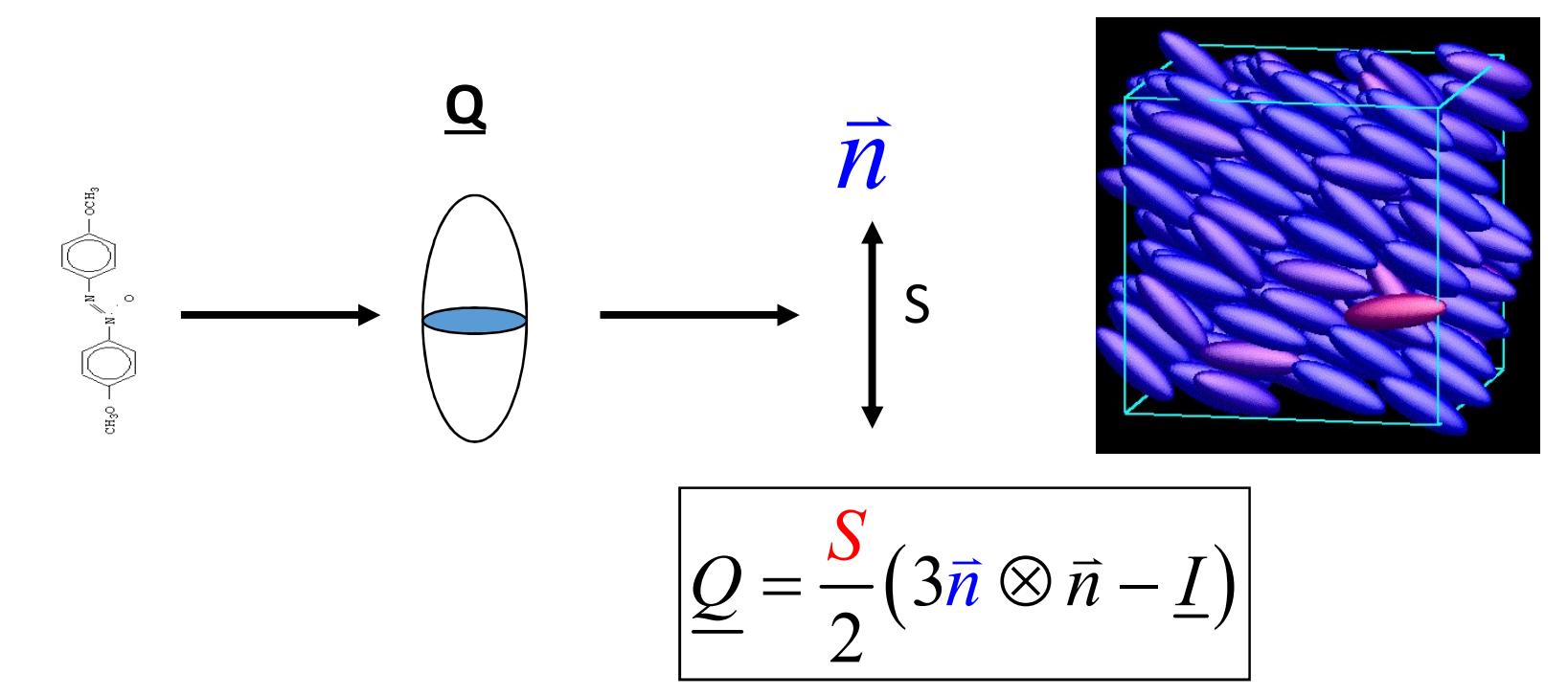

\section{Translational order :}

$$
\begin{aligned}
& \psi=\eta e^{i \phi} \\
& \text { e.g. : } \phi=q_{0} z
\end{aligned}
$$



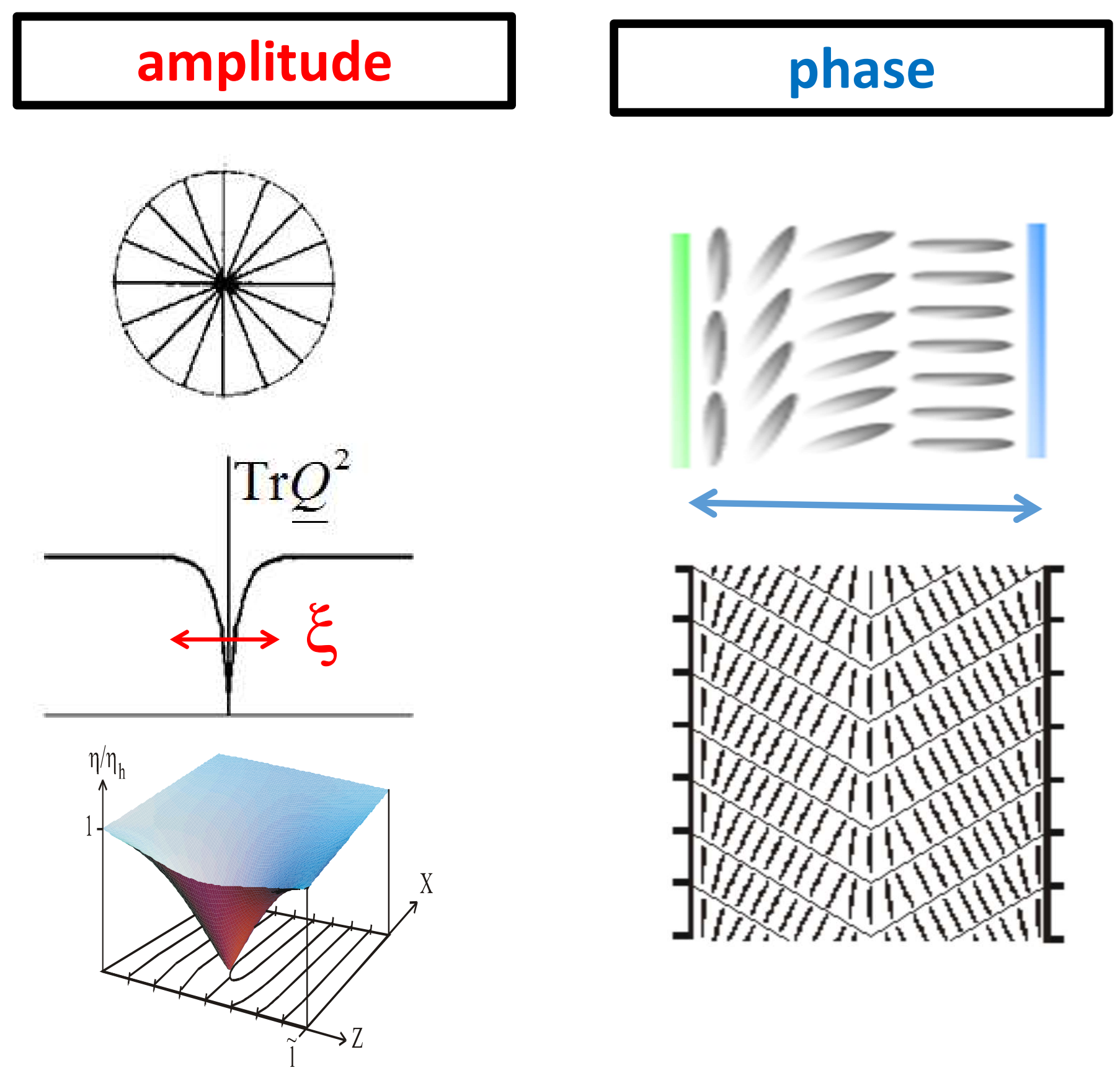

Crystals 


\section{Topological charge}

\section{Order parameter space}

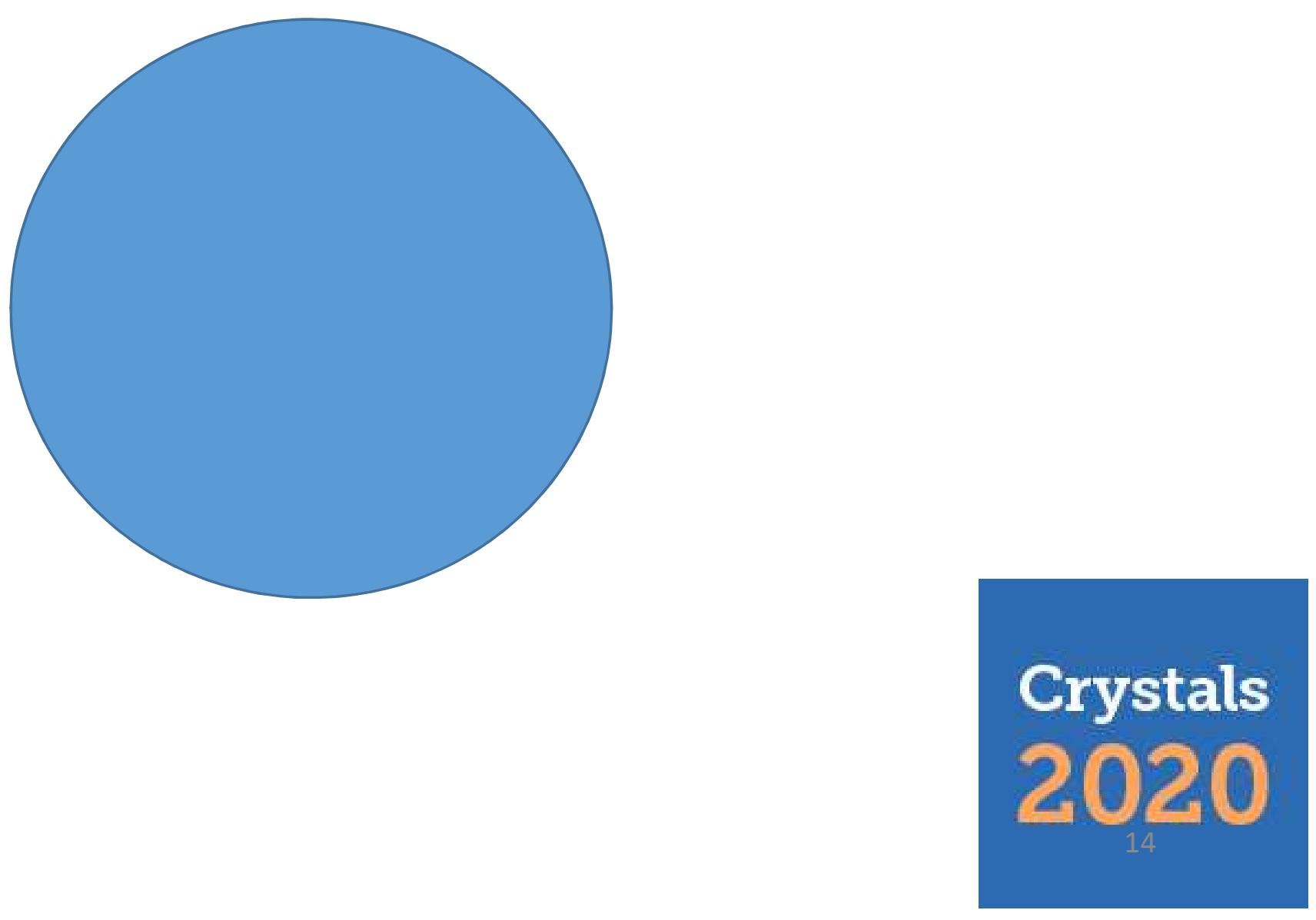




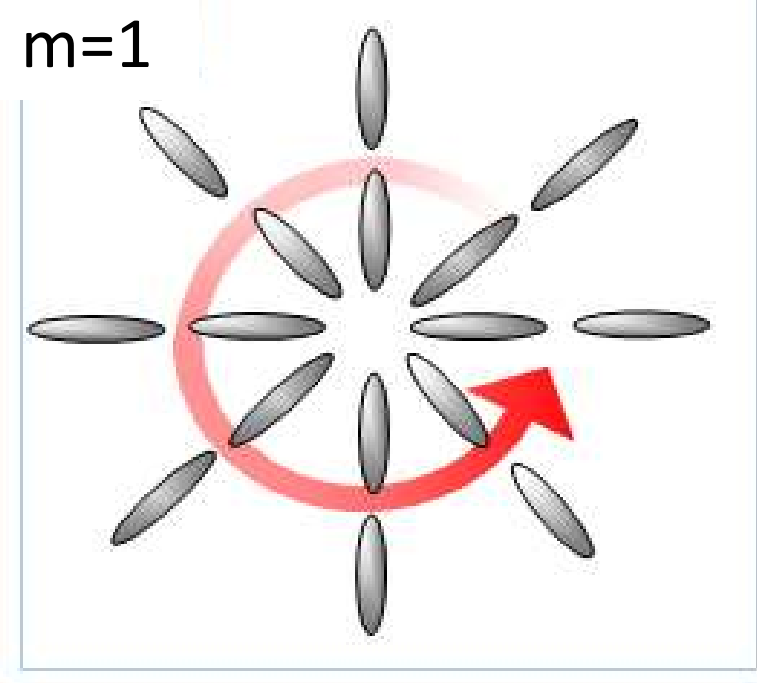

defect

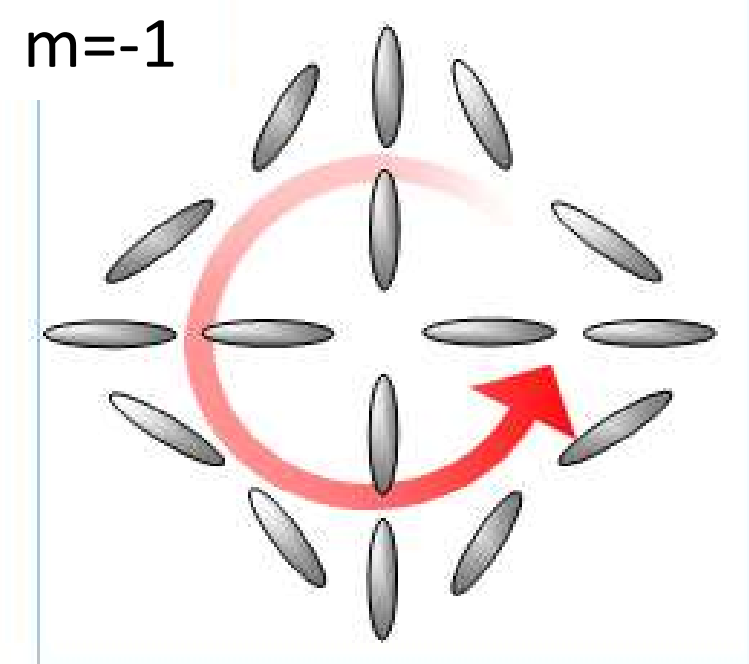

anti-defect

$$
\begin{array}{|l|l|}
\sum_{\text {encircle }} \theta_{\text {rotation }} & \begin{array}{l}
m \in\{ \pm 1 / 2, \pm 1, \pm 3 / 2 \ldots . .\} \\
2 \pi
\end{array} \\
\text { Conserved quantity }
\end{array}
$$




\section{Origin of TDs}

Continuous symmetry breaking

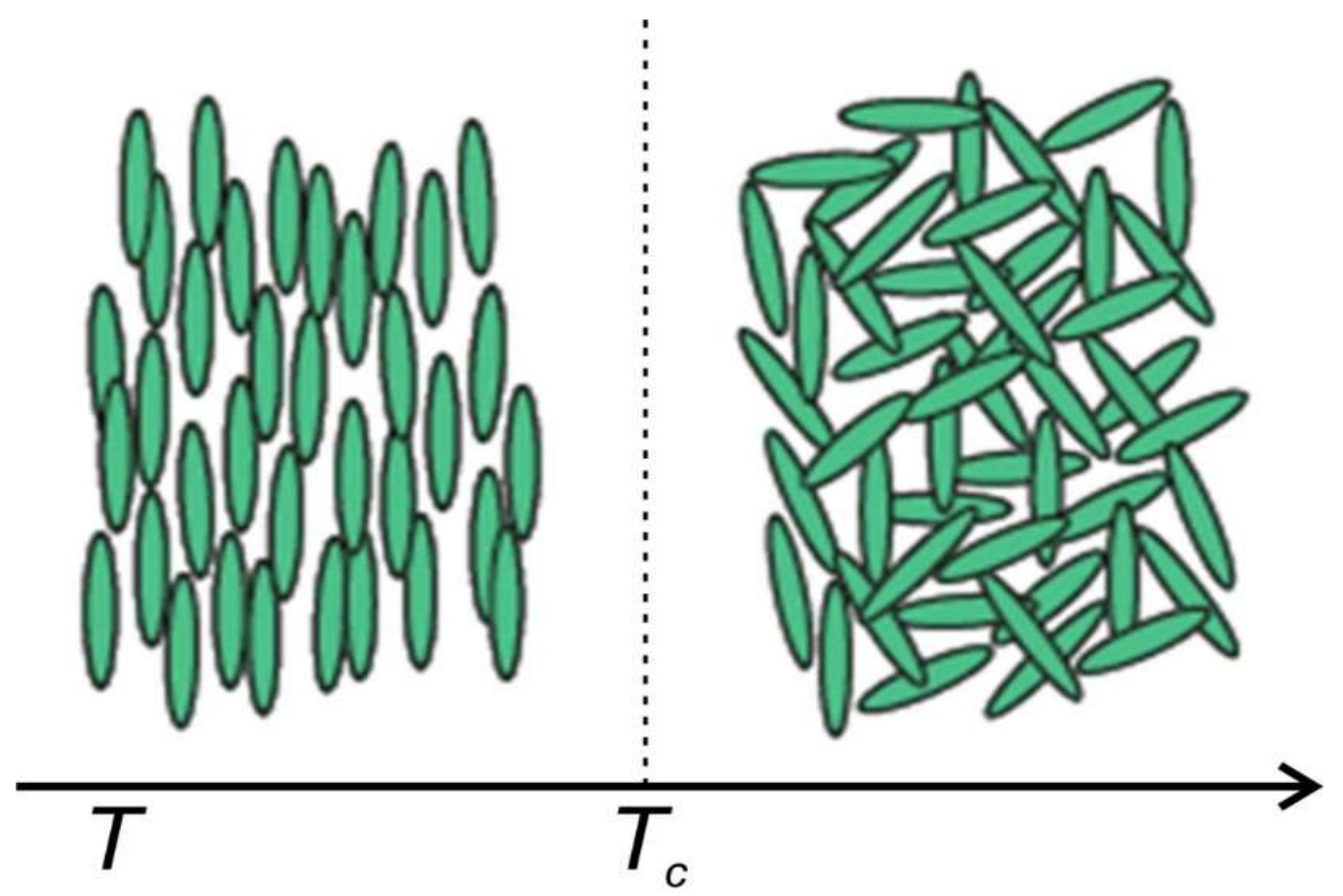

Crystals 

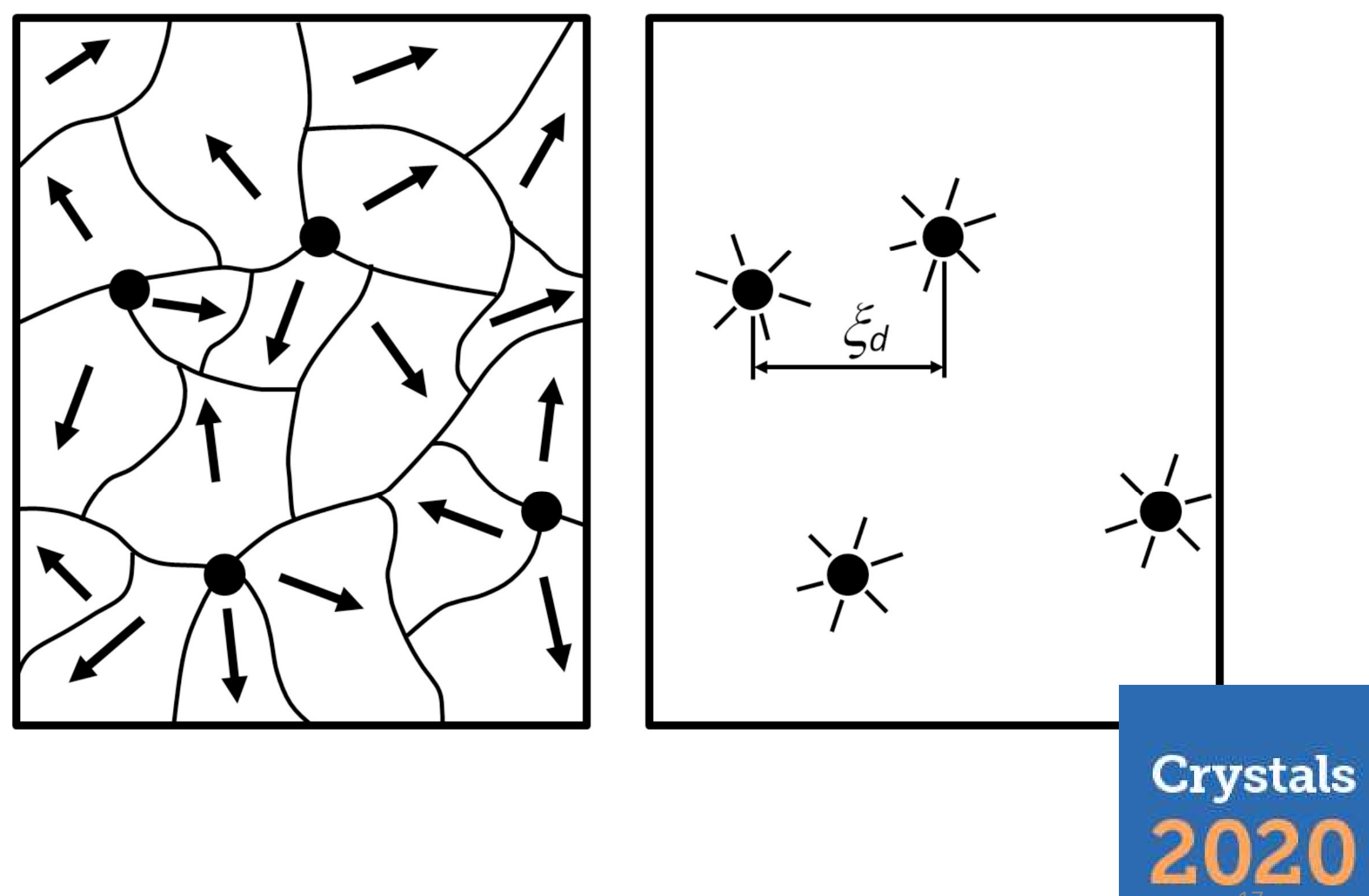


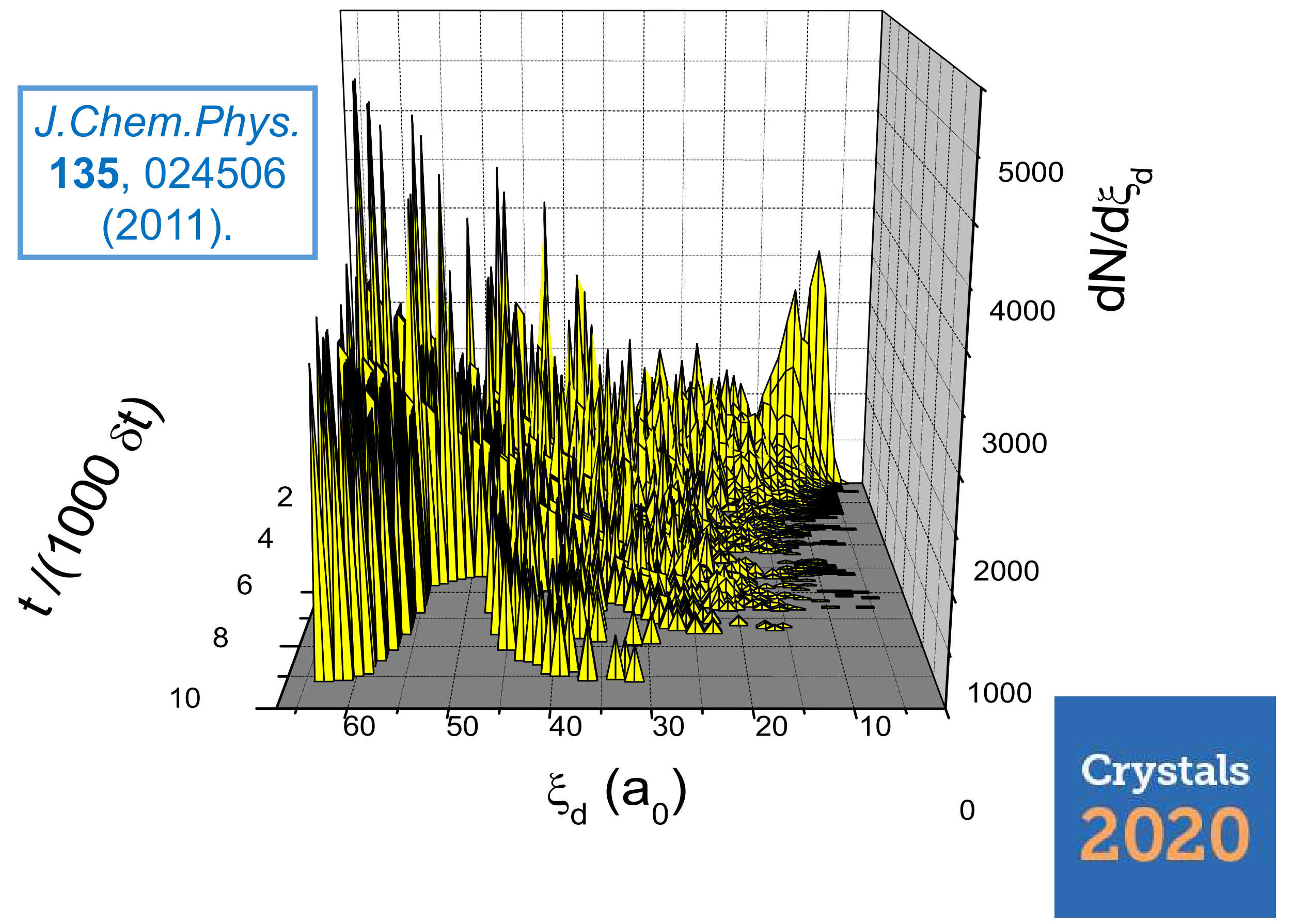



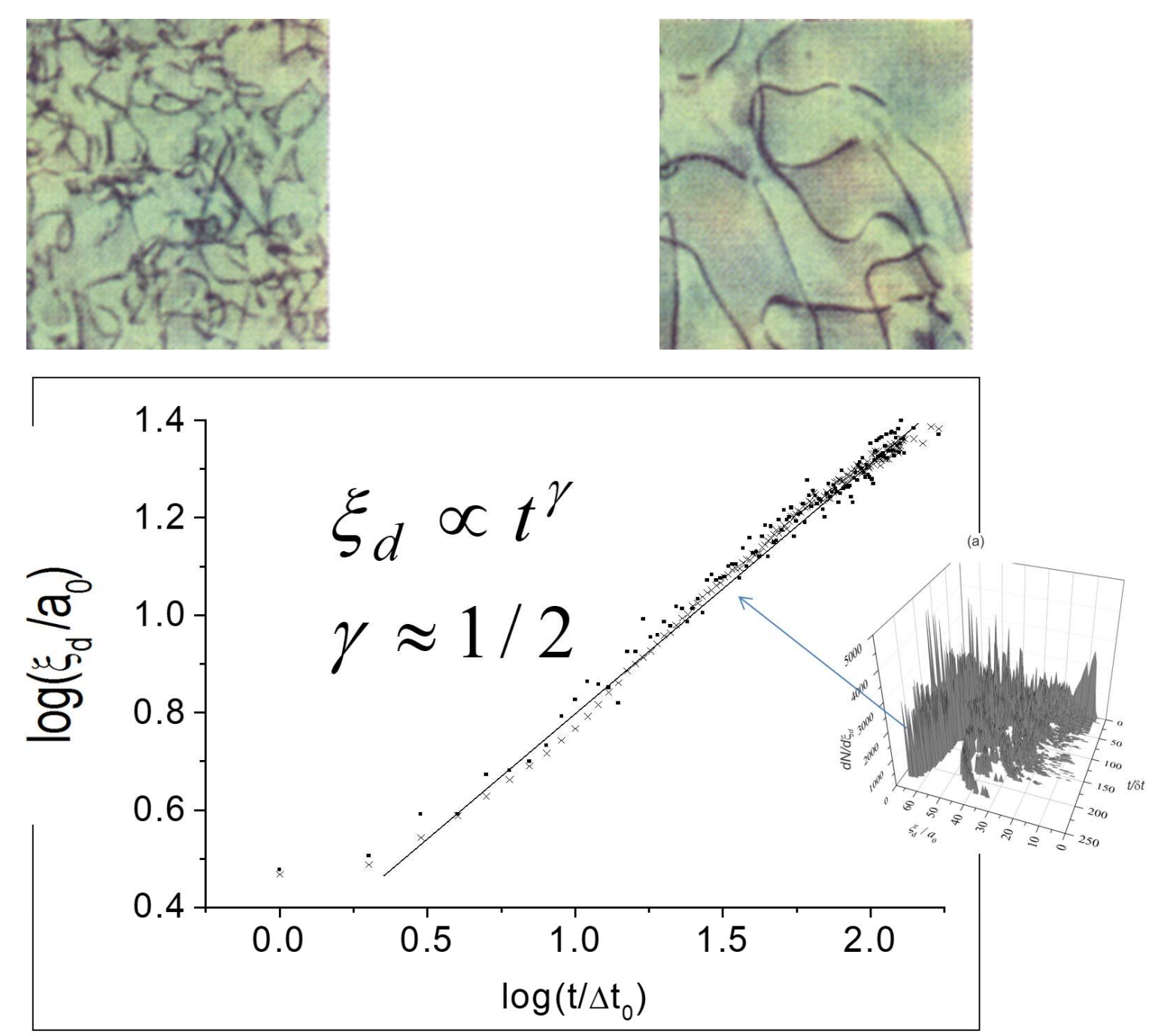

Phys.Rev.E 65, 021705 (2002). J.Chem.Phys. 135, 024506 (2011). 


\section{Stabilisation : confinement}

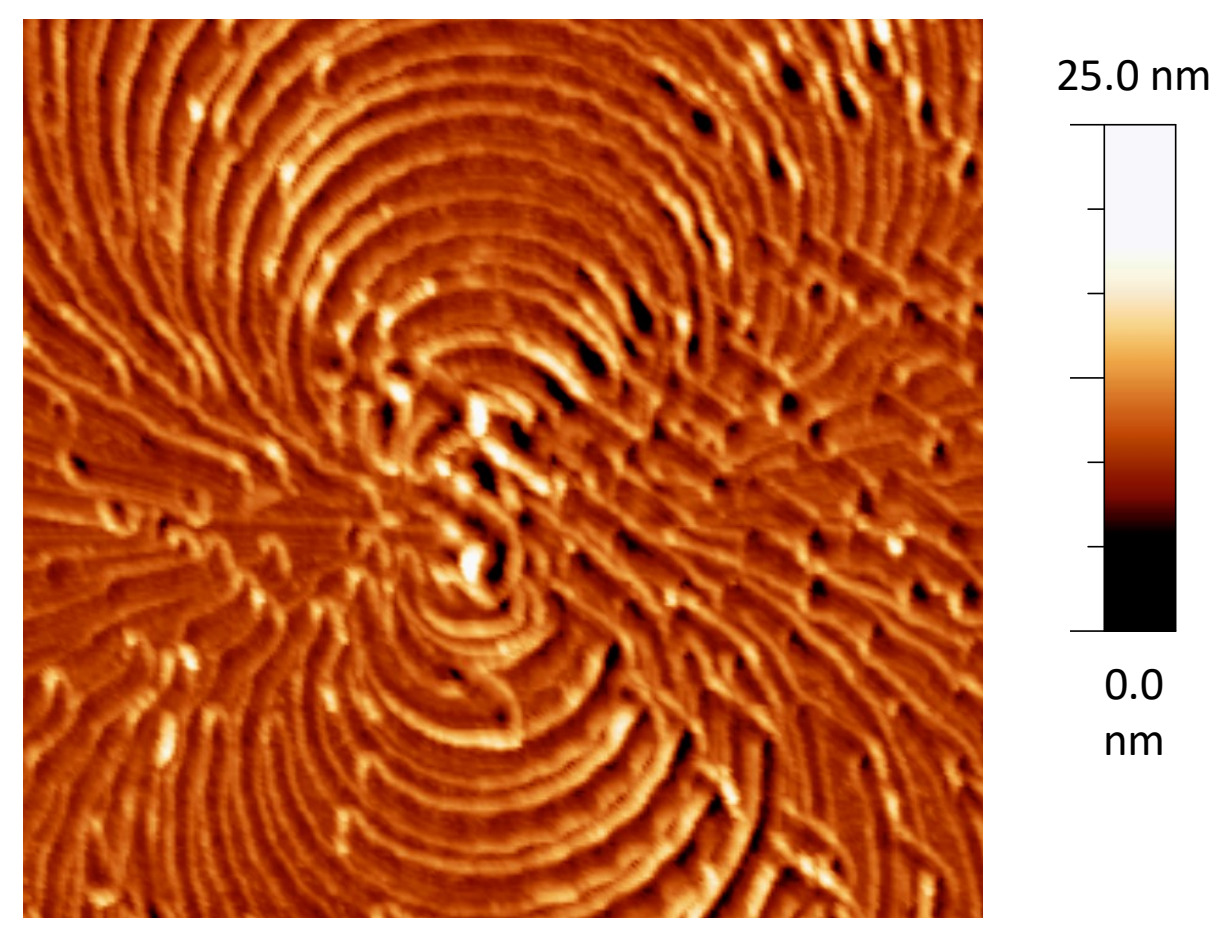

$$
\theta=\sum_{i=1}^{N}\left(m_{i} \operatorname{ArcTan}\left(\frac{y-y_{i}}{x-x_{i}}\right)+\theta_{0}^{(i)}\right)
$$

Phys. Rev. E 95, 042702 (2017) 

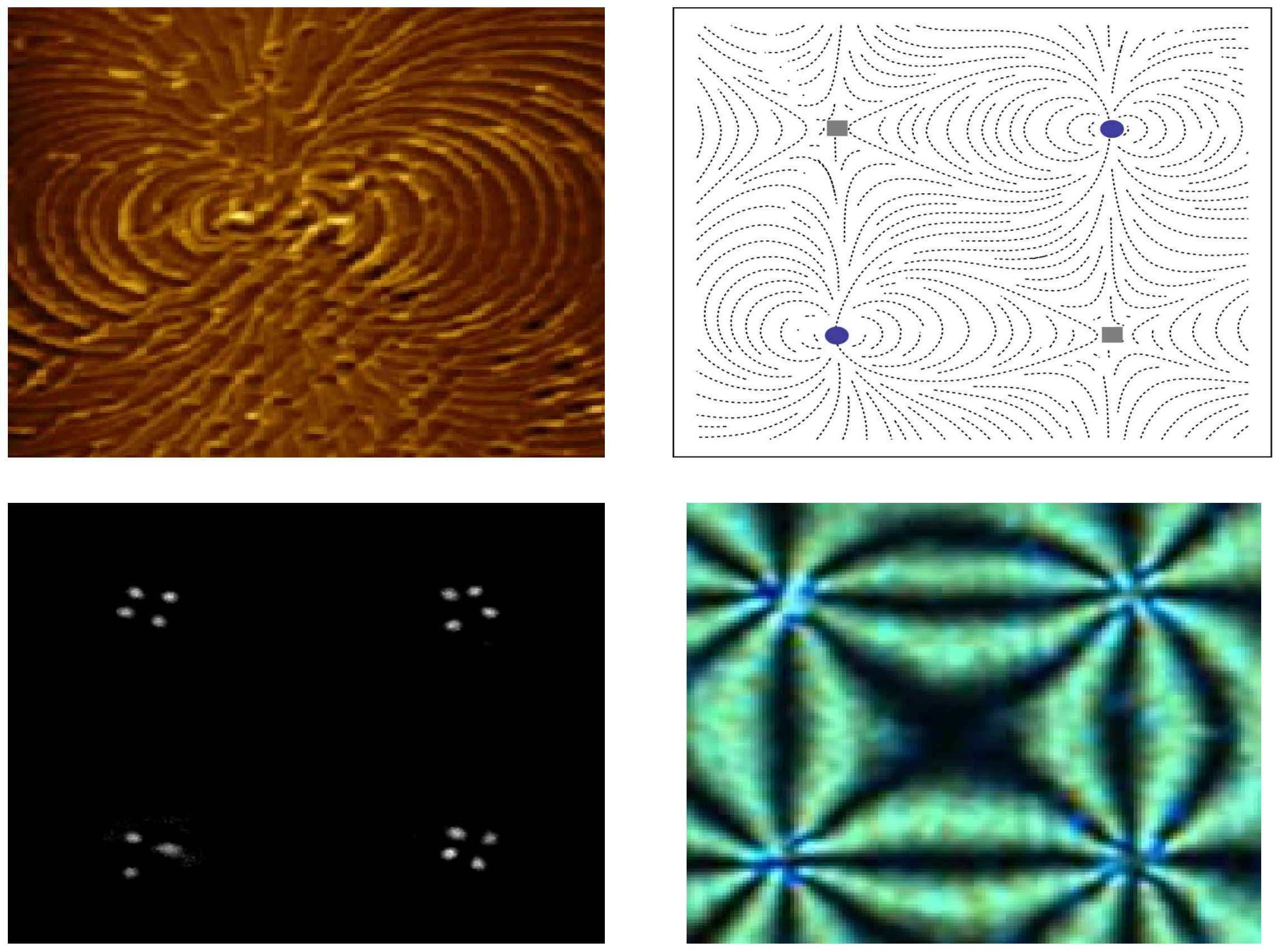
(a)
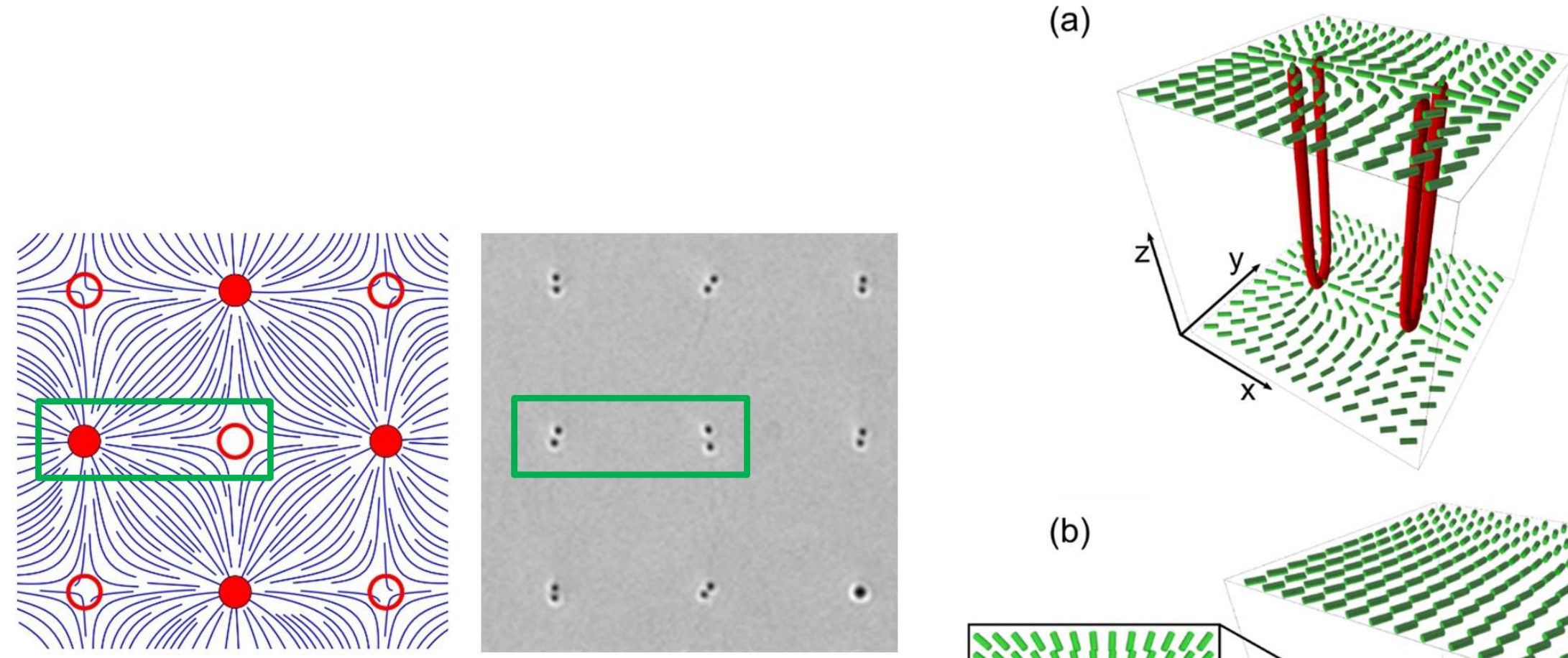
$m=+1$
O $=-1$

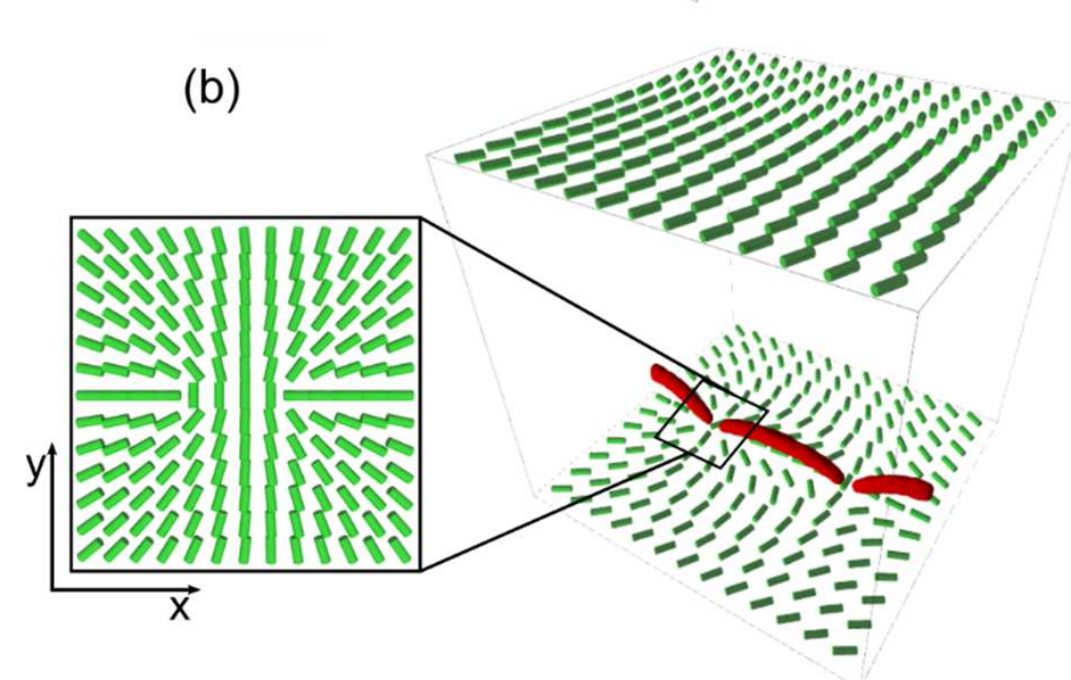

Crystals 2020 
(a)

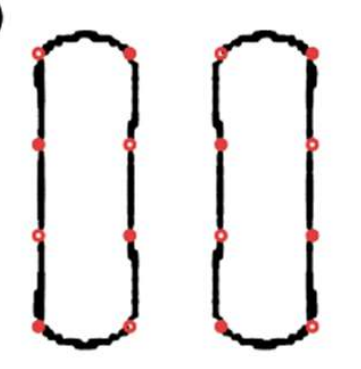

(b)
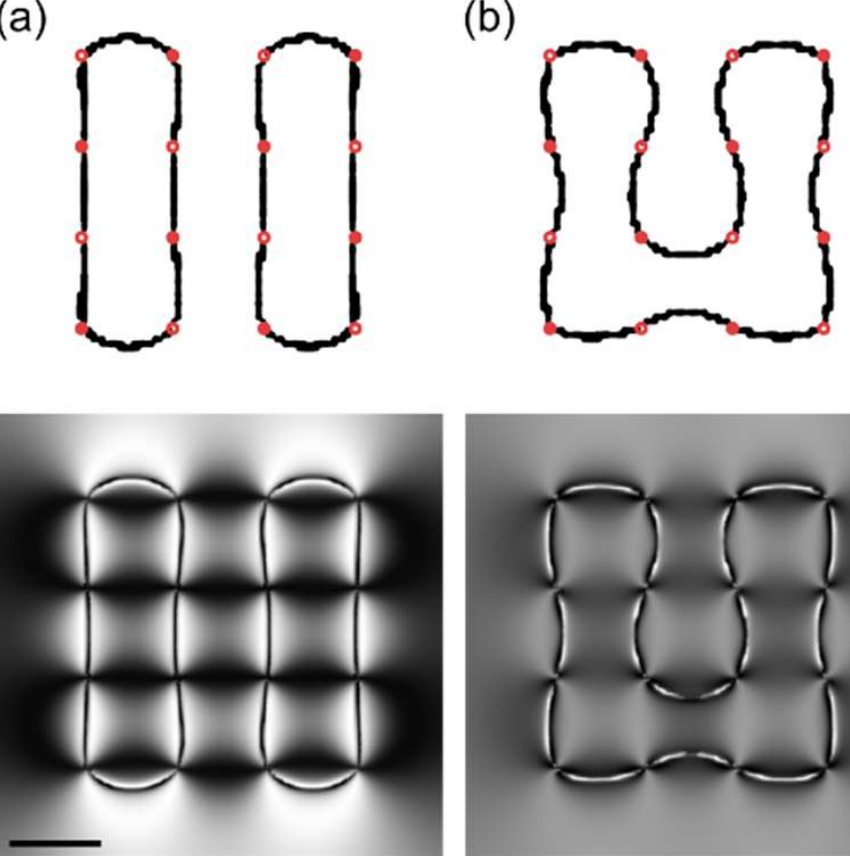

(d)

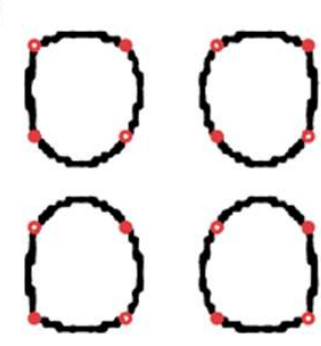

e)

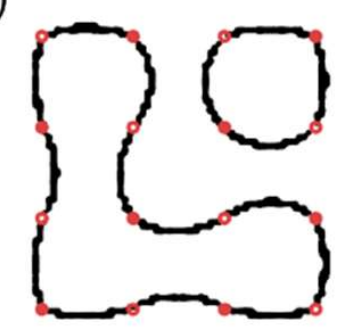

(f)
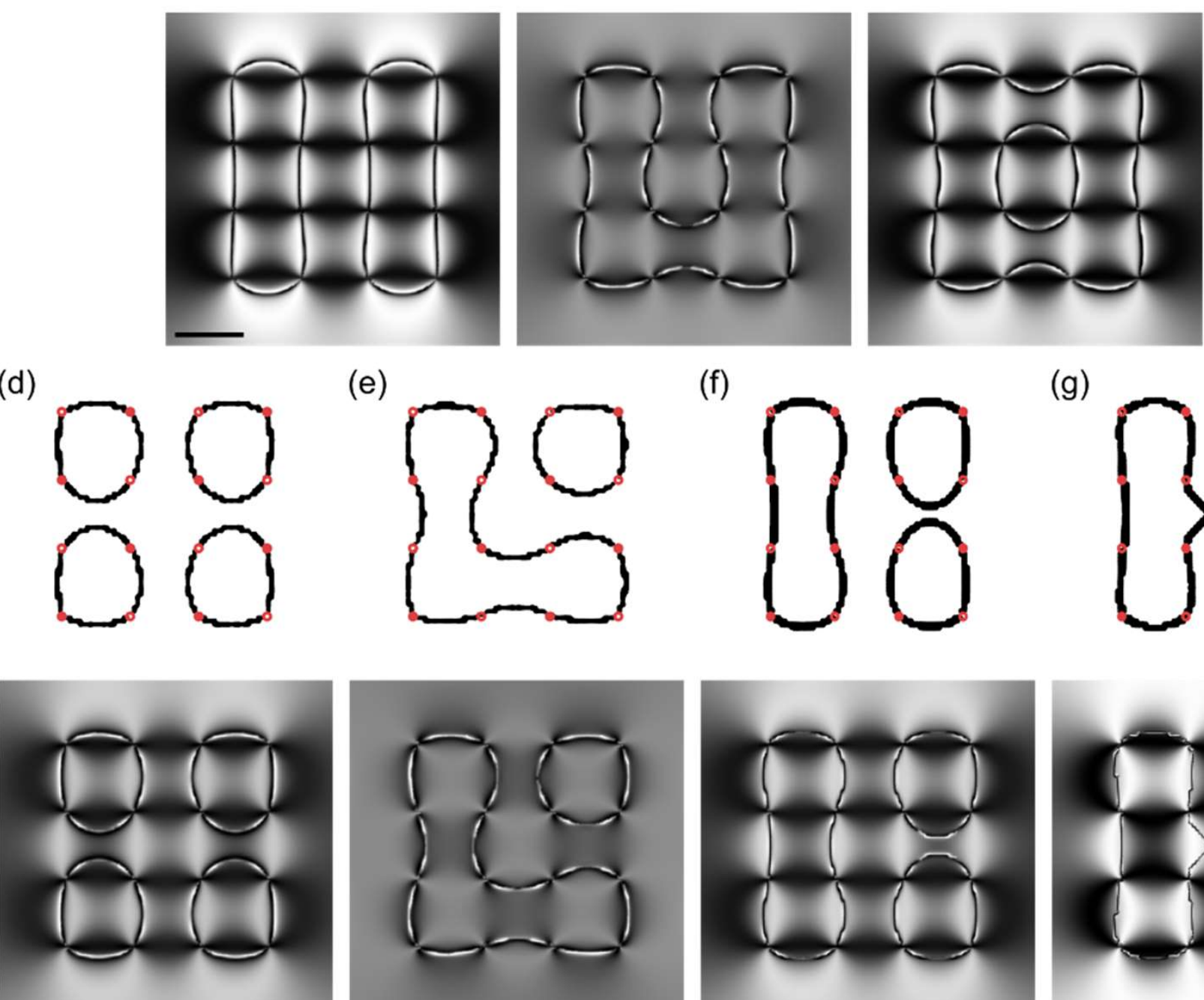

(g)
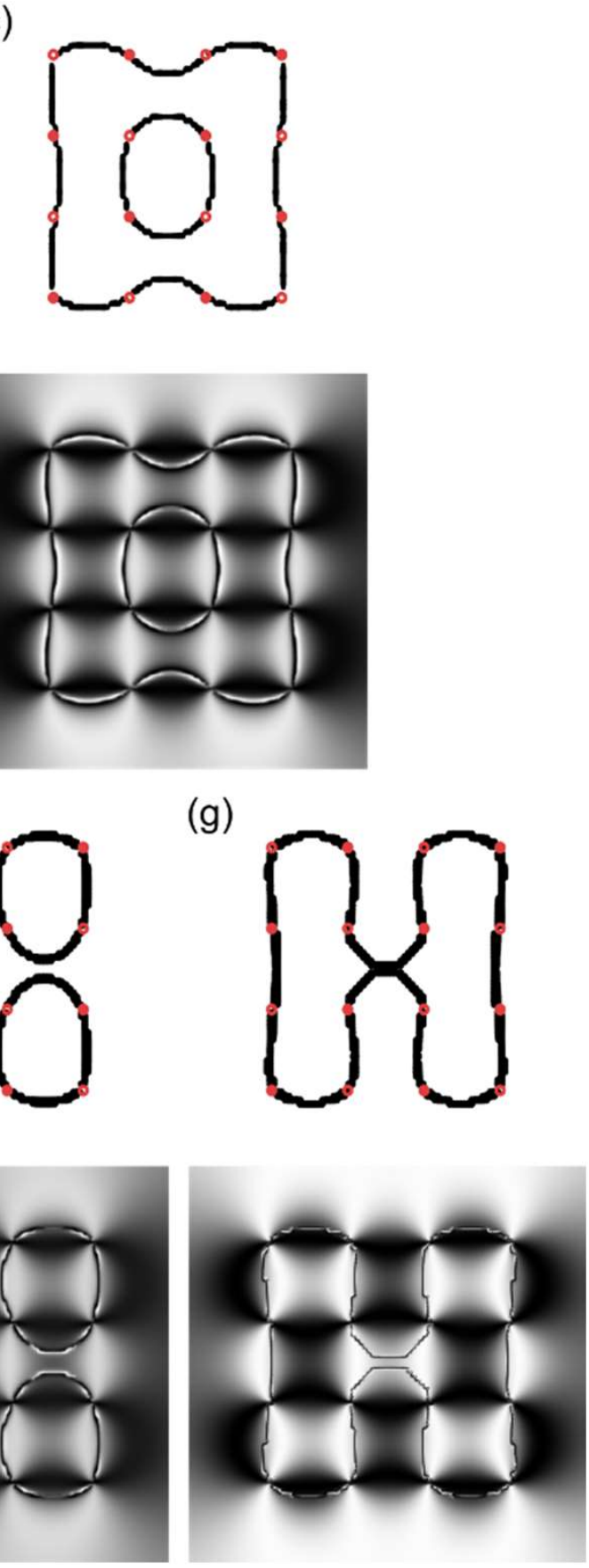

Crystals 2020 

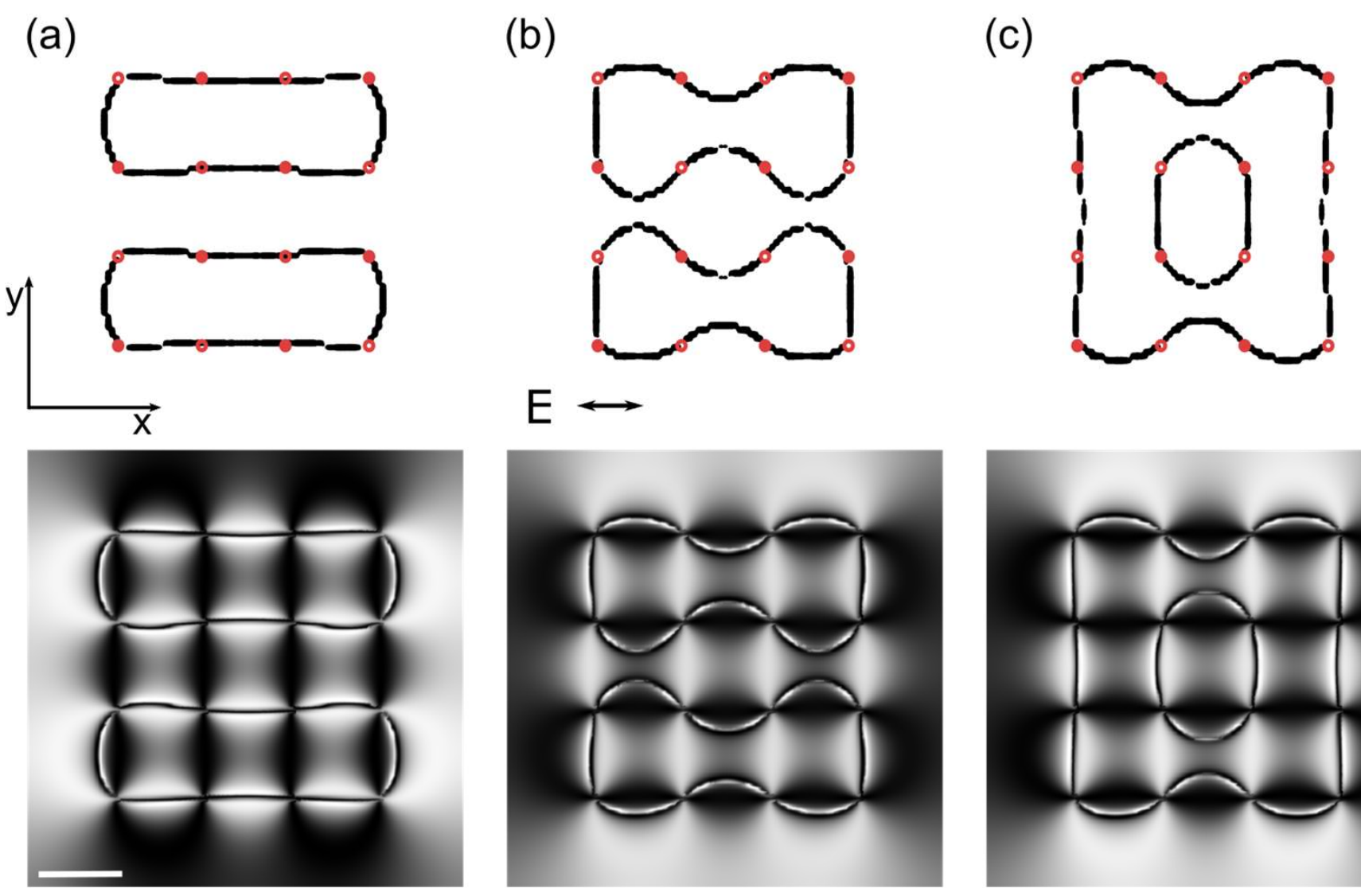

(d) $\left\{\begin{array}{cc}1 & 1 \\ 1 & 1 \\ 1 & 1 \\ 1 & 1\end{array}\right\}$
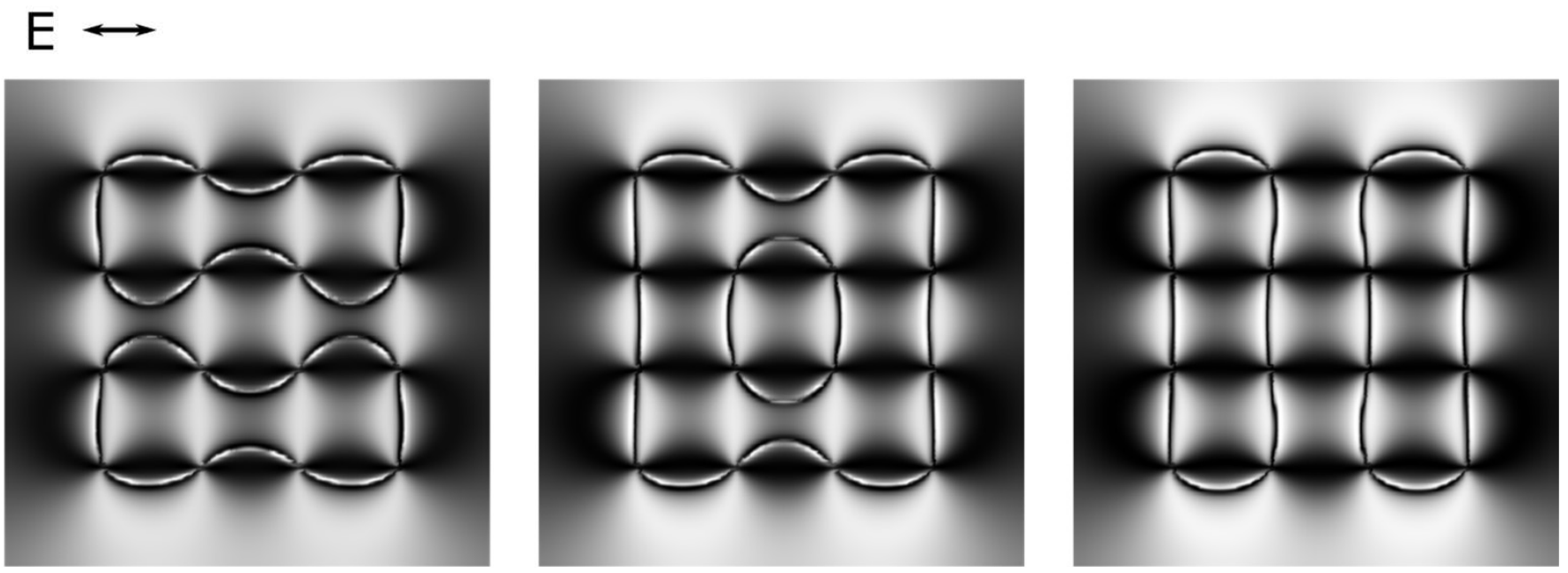

Phys. Rev. Res. 2, 0131761 (2020). 


\section{Stabilisation : curvature}

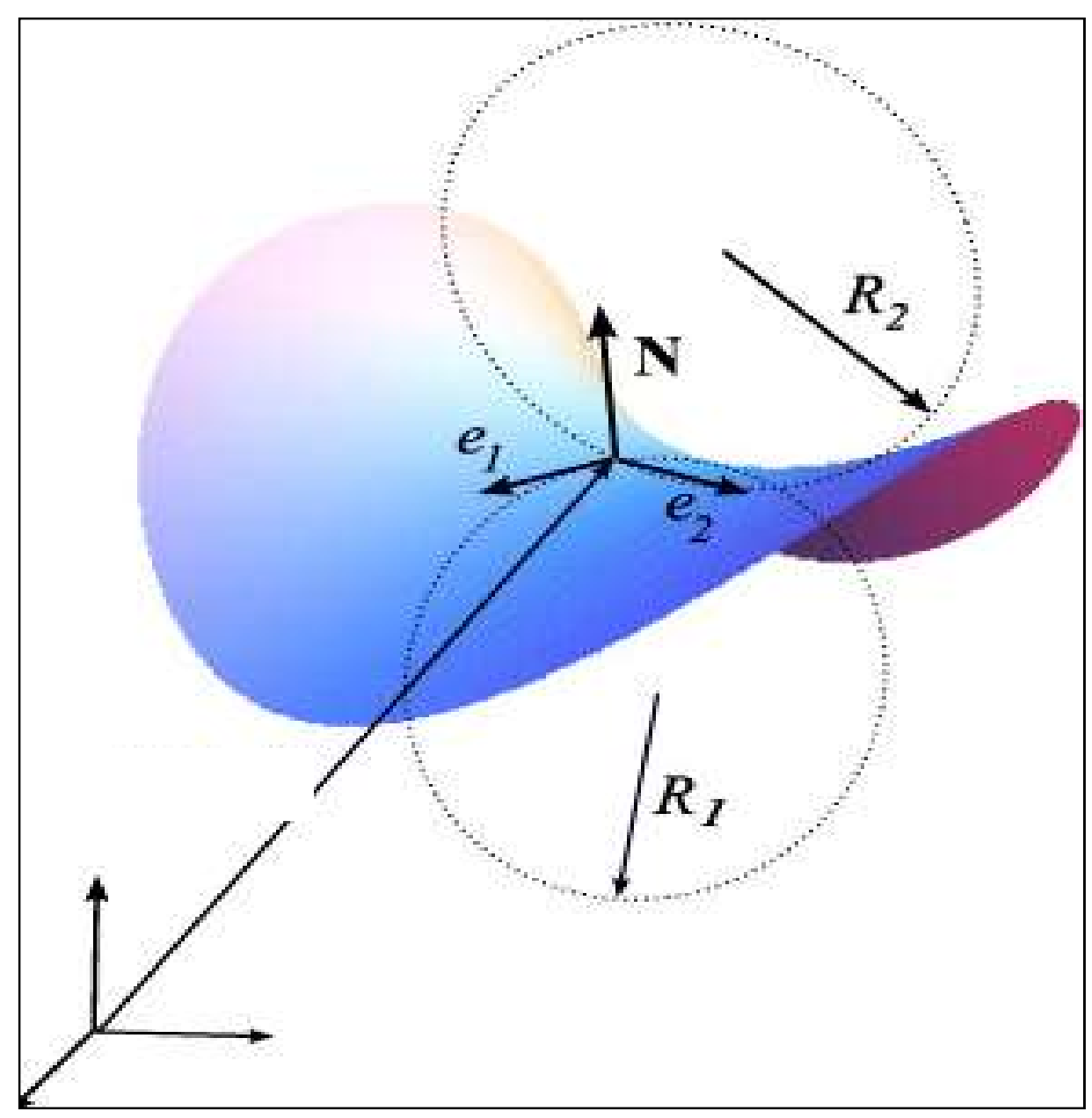

\section{Gaussian curvature}

$$
K=C_{1} C_{2}=\frac{1}{R_{1} R_{2}}
$$




\section{Gauss - Bonnet \& Poincaré-Hopff theorem}

$$
m_{t o t}=\frac{1}{2 \pi} \oiint K d^{2} \vec{r}=\chi
$$

\begin{tabular}{|l|l|l|}
\hline \multicolumn{2}{|c|}{ Geometry } & $\chi$ \\
\hline Sphere & & 2 \\
\hline Torus & & 0 \\
\hline Double torus & & -1 \\
\hline
\end{tabular}




\section{Stabilisation : curvature}

Effective Topological Charge Cancelation (ETCC) mechanism

$$
\Delta m_{e f f}(\Delta \zeta)=\frac{1}{2 \pi} \iint_{\Delta \zeta} K d^{2} \vec{r}
$$

$$
\Delta m_{e f f}(\Delta \zeta) \rightarrow 0
$$

Sci. Rep. 6, 27117 (2016).

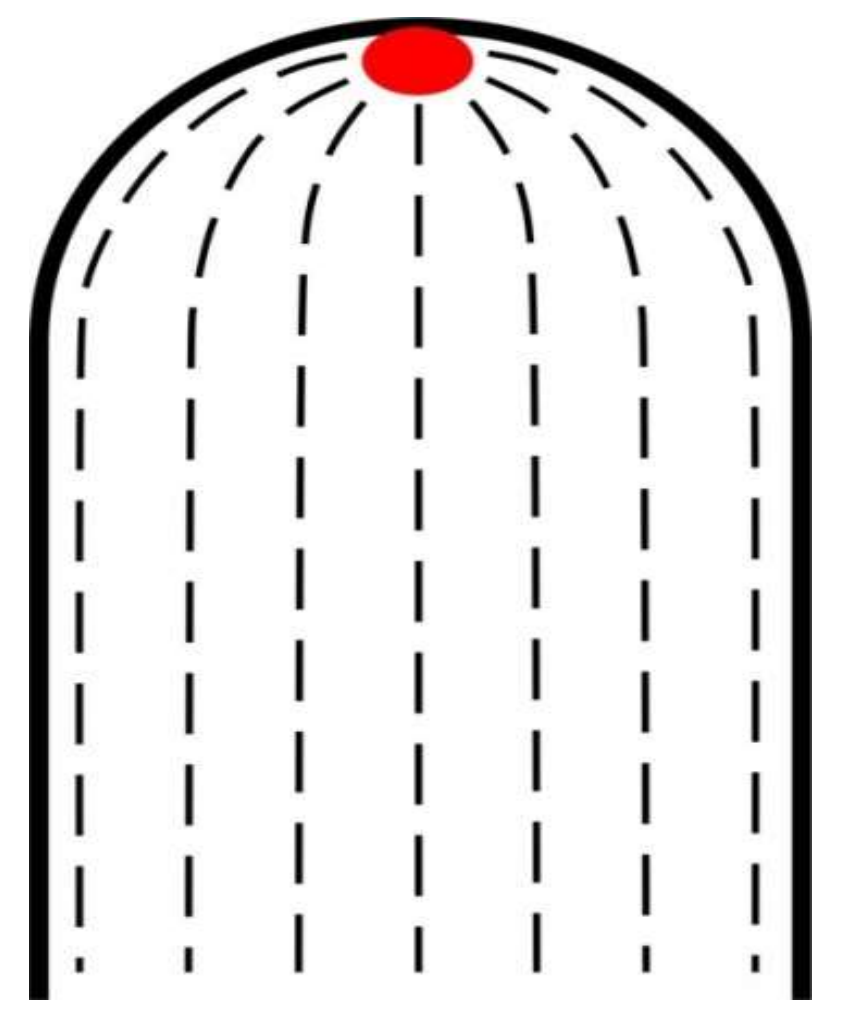




\section{TORUS}

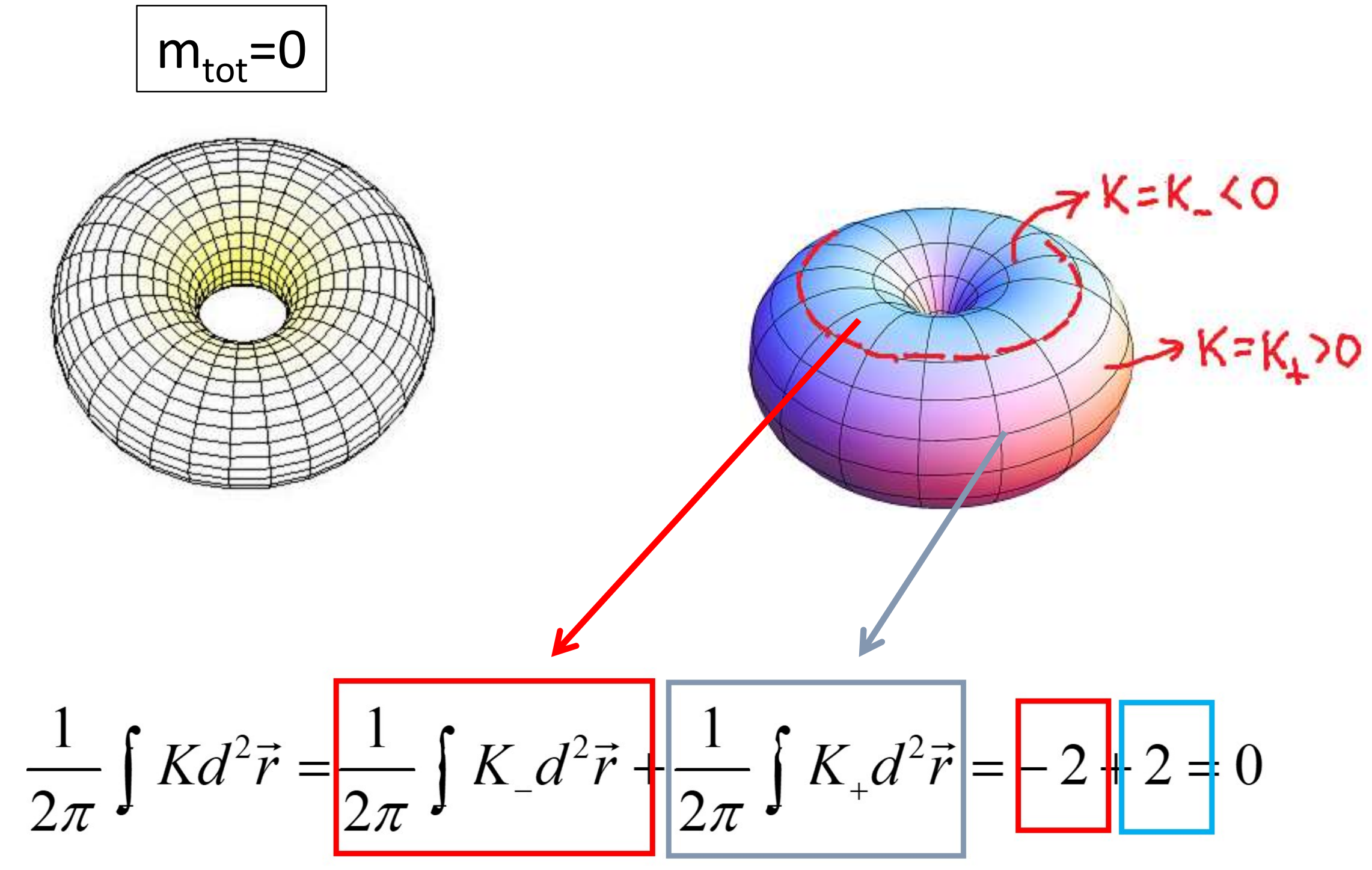



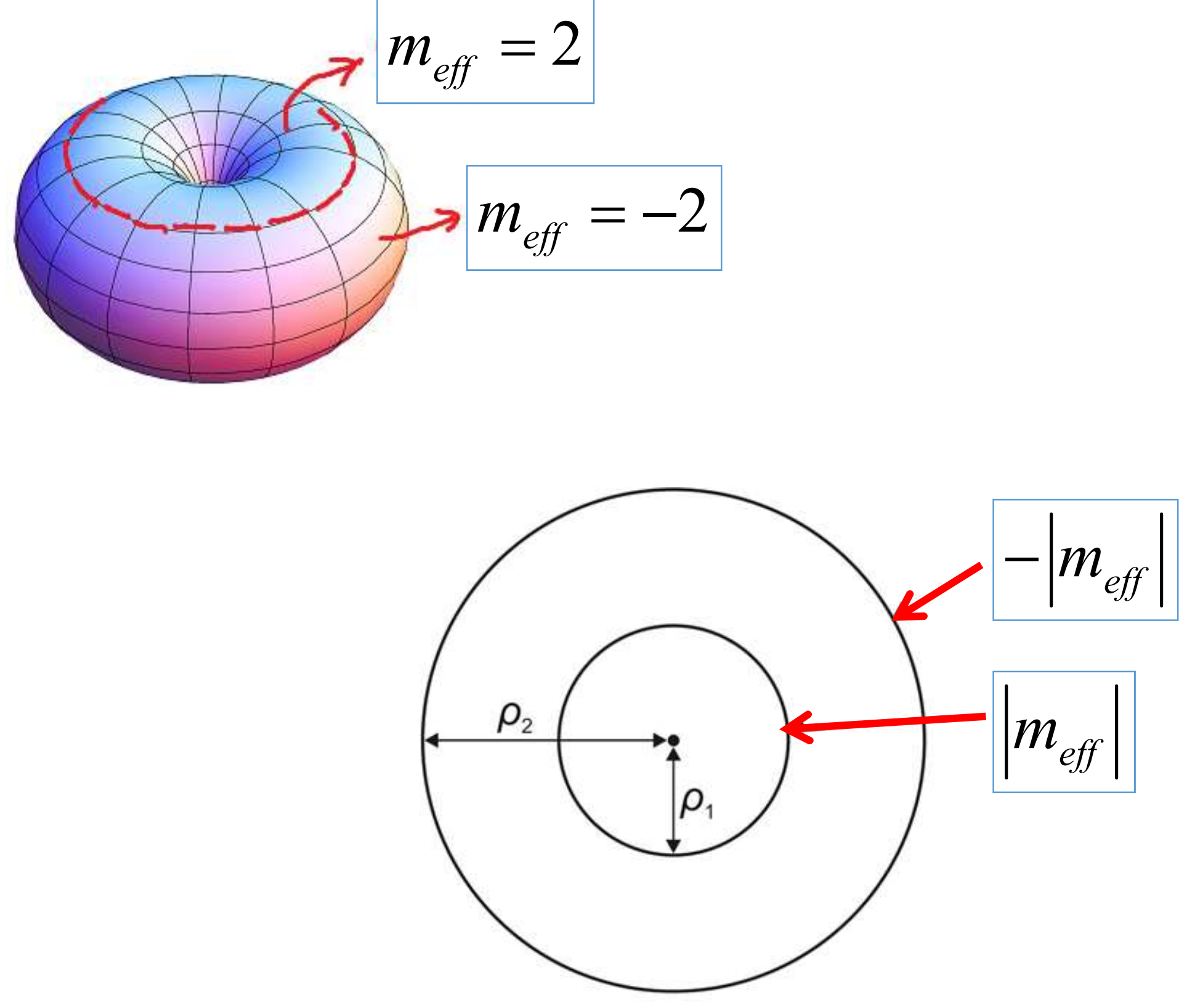

Crystals 2020 


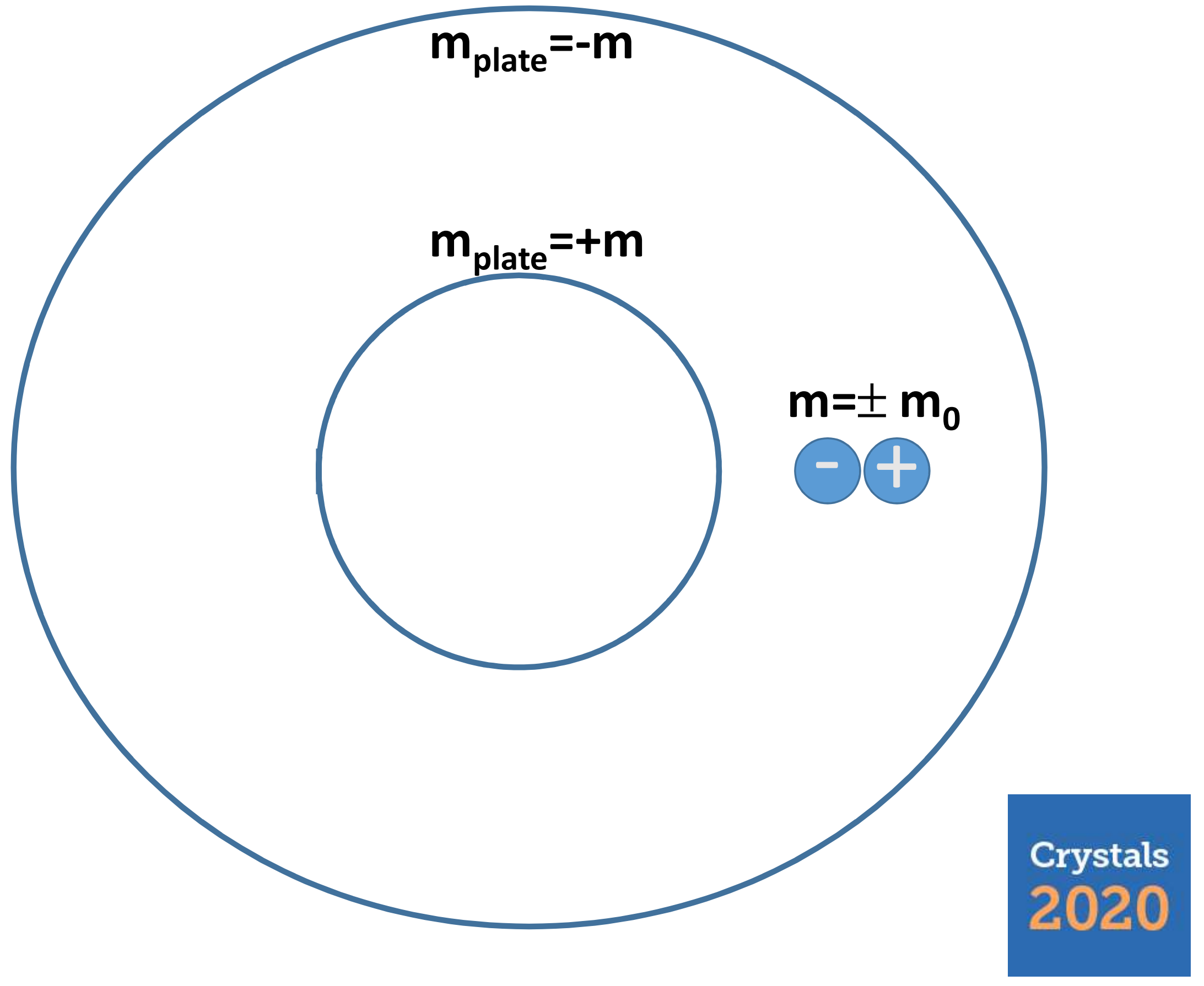




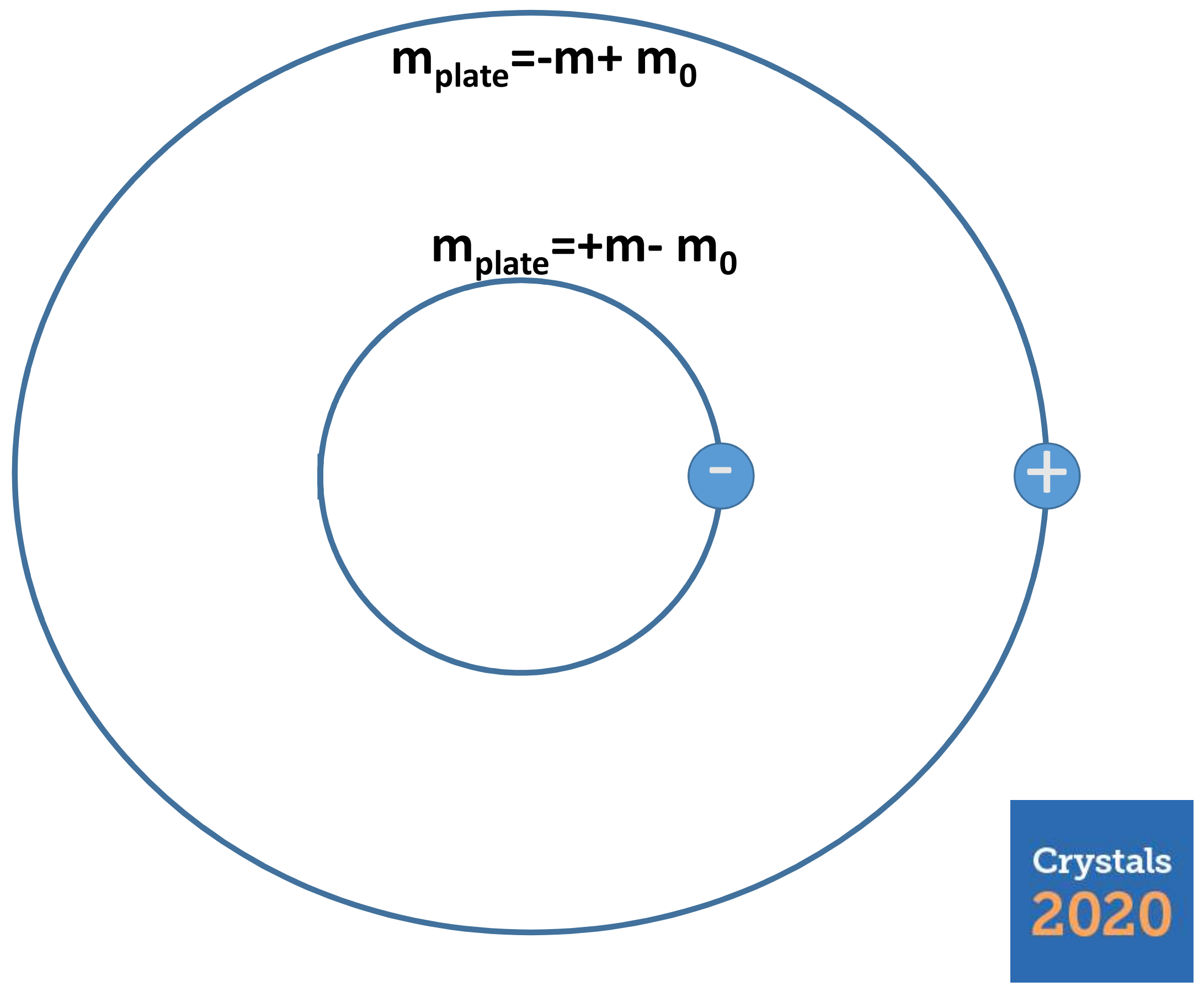




\section{D electrostatics :}

$$
\begin{gathered}
W_{p}=\frac{e_{1} e_{2}}{2 \pi \varepsilon_{0} h} \ln \rho+\text { const } . \\
F_{12}=E_{1} e_{2}=\frac{1}{2 \pi \varepsilon_{0} h} \frac{e_{1} e_{2}}{\rho} \\
E=\frac{1}{2 \pi \varepsilon_{0} h} \frac{e}{\rho}
\end{gathered}
$$$$
1+\frac{1}{2}\left(\ln \left(\frac{\rho_{2}}{\rho_{1}}-1\right) \frac{\rho_{1}}{2 \xi}\right) \sim \Delta m_{e f f} \ln \left(\frac{\rho_{2}}{\rho_{1}}\right)
$$

\section{D nematic :}

$$
W_{\mathrm{int}} \sim m^{2} 4 \pi K_{f} \ln \left(\frac{\rho}{a_{0}}\right)
$$$$
F_{12}=E m \sim 4 \pi K_{f} \frac{m^{2}}{\rho}
$$

$$
E \sim 4 \pi K_{f} \frac{m}{\rho}
$$

$$
\text { penalty = gain }
$$



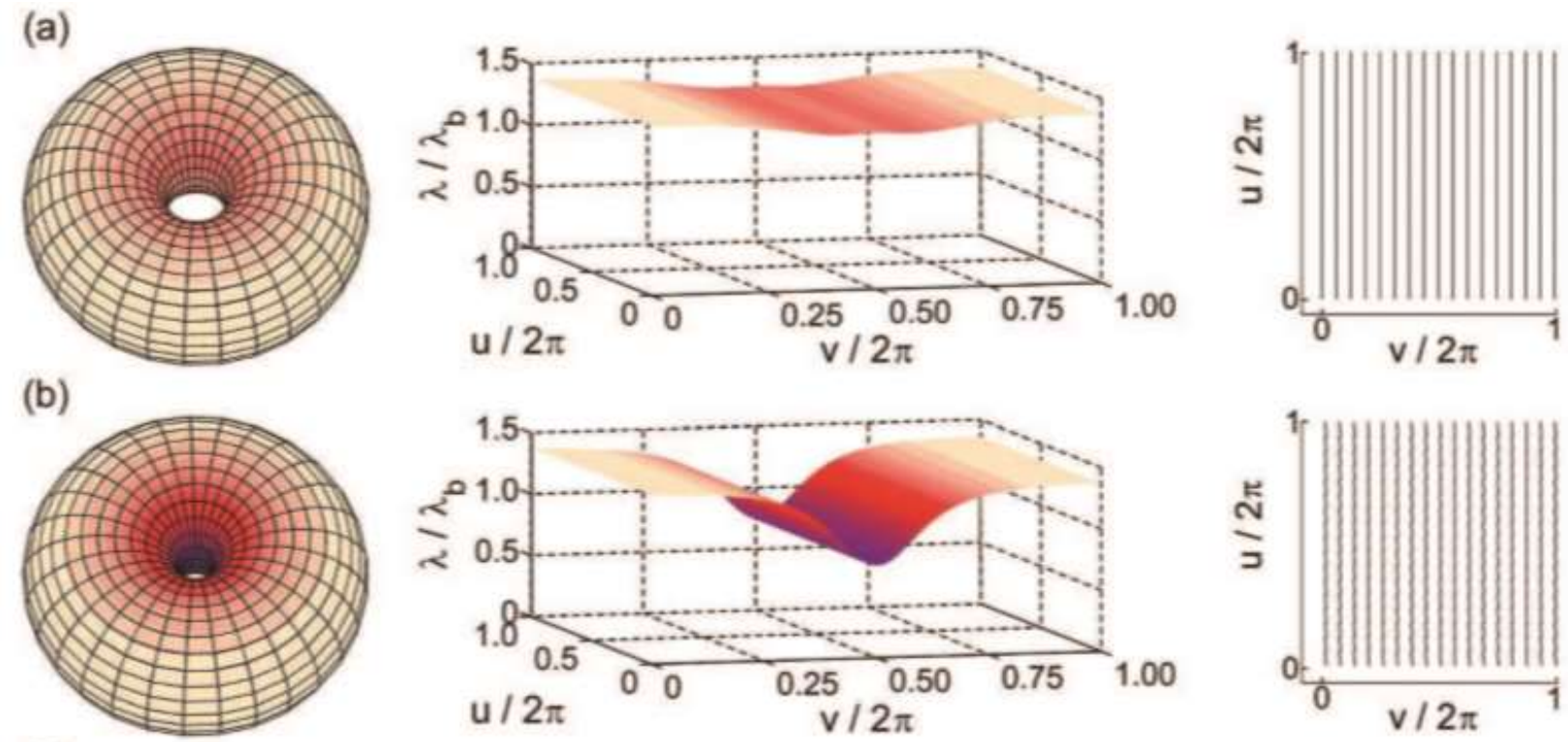

(c)
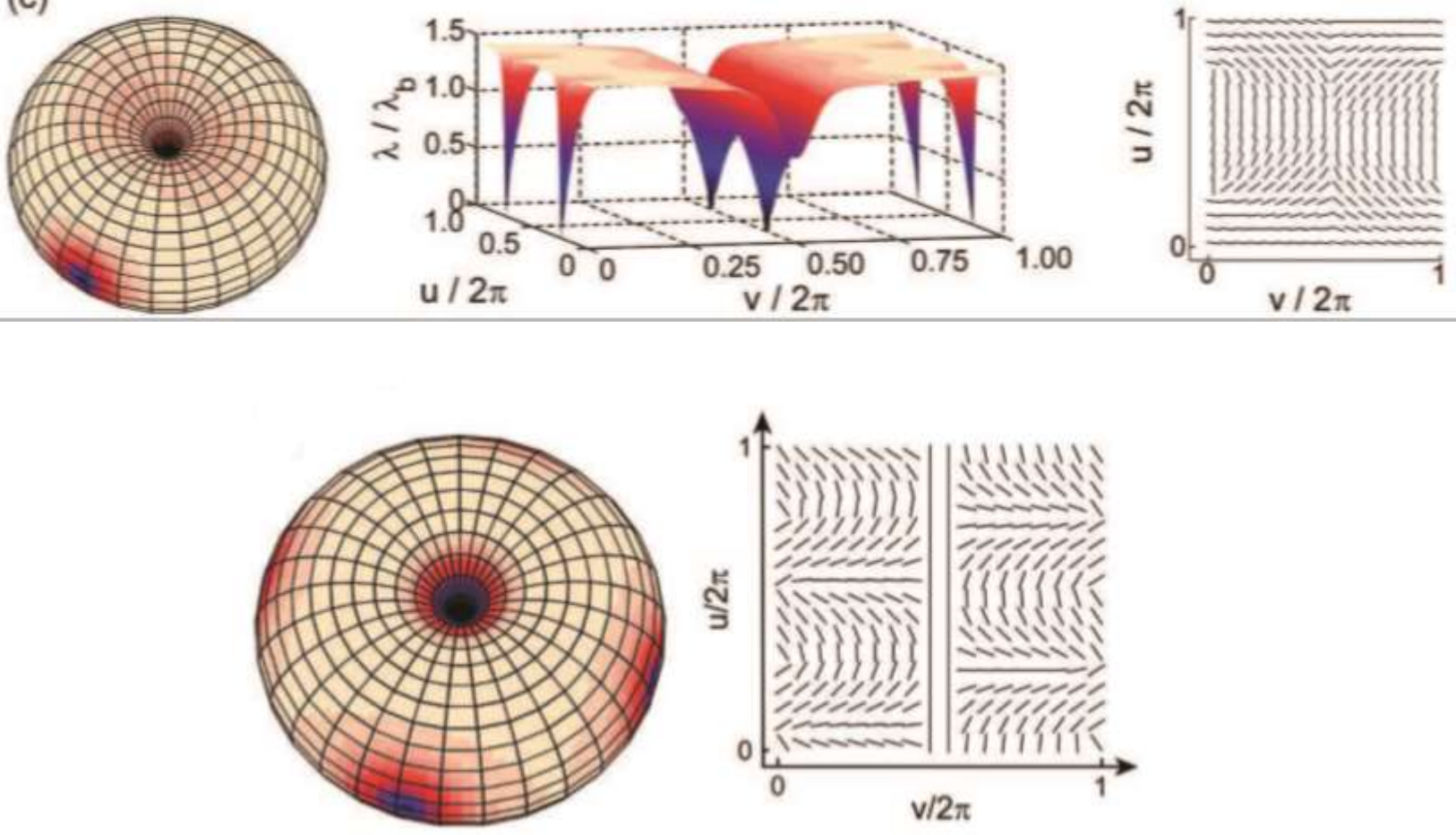

Crystals 2020 


\section{Intrinsic and extrinsic curvature}

\section{Key curvature terms}

$$
\begin{aligned}
& W_{\text {int }}=-J \sum_{i, j(n n)} \vec{S}_{i} \vec{S}_{j} \mid \\
& W_{\text {int }}=\iint \frac{K}{2}|\nabla \vec{S}|^{2} d A \quad \vec{S}=\vec{e}_{1} \cos \theta+\vec{e}_{2} \sin \theta \\
& |\nabla \vec{S}|^{2}=|\nabla \theta-\vec{A}|^{2}+\left|\begin{array}{c}
\vec{e}_{1} \cdot \underline{C}^{2} \vec{e}_{1} \\
\text { Extrinsic }
\end{array} \quad \underline{C}=\right| \begin{array}{cc}
1 / R_{1} & 0 \\
0 & 1 / R_{2}
\end{array} \mid
\end{aligned}
$$


Variational parameters: $\underline{Q}, \underline{C}$

Vary relative volume :

$$
v=V / V_{0}
$$
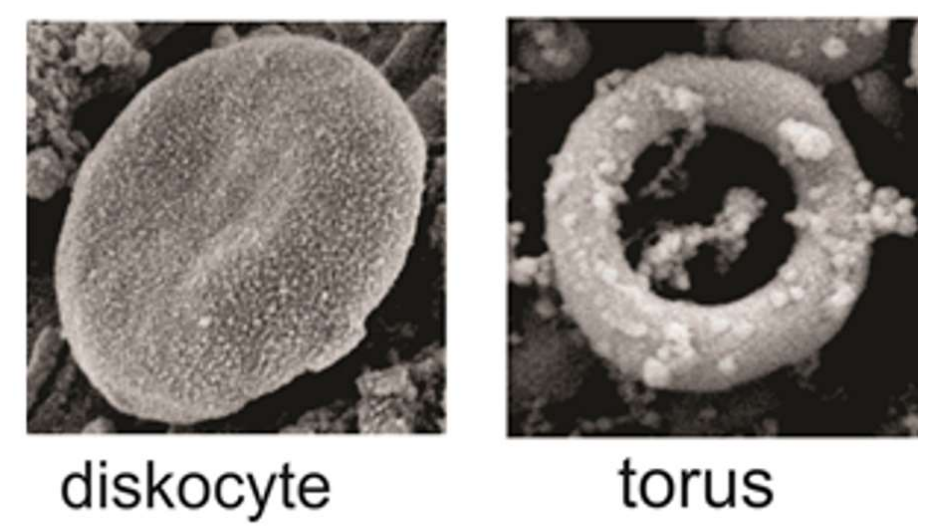

$$
\begin{gathered}
S=4 \pi R_{0}^{2} \\
V_{0}=4 \pi R_{0}^{3} / 3
\end{gathered}
$$
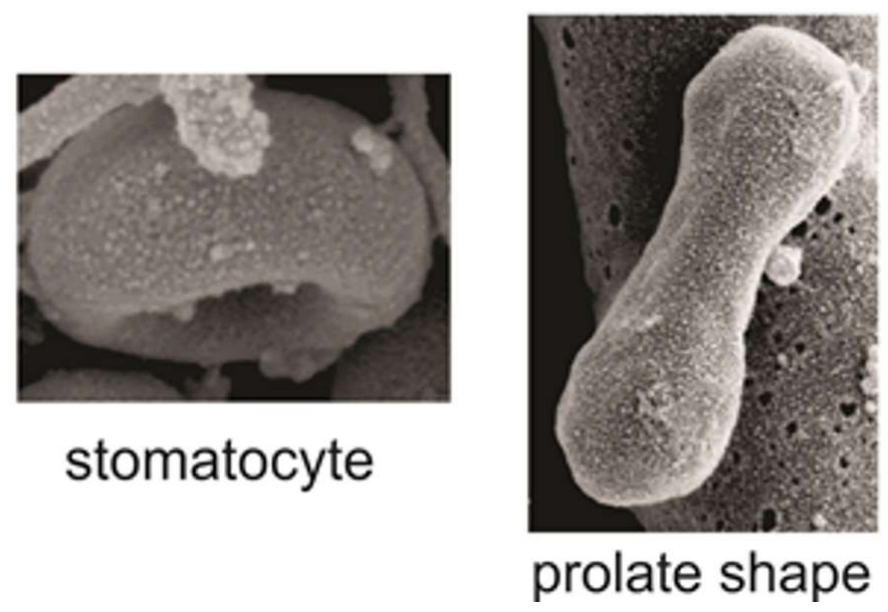


\section{Intrinsic curvature term}

$$
K|\nabla \theta-\vec{A}|^{2} \rightarrow 0
$$

$$
\nabla \theta=\vec{A}
$$

$$
\operatorname{curl} \nabla \theta \equiv 0 \neq \operatorname{curl} \vec{A} \propto K
$$




$$
f^{(\text {intrinsic })}=K_{e}|\nabla \theta-\vec{A}|^{2}
$$

$$
\nabla \times \nabla \theta=0 \neq \nabla \times \vec{A} \propto K_{g}=\frac{1}{\boldsymbol{R}_{1} \boldsymbol{R}_{2}}
$$

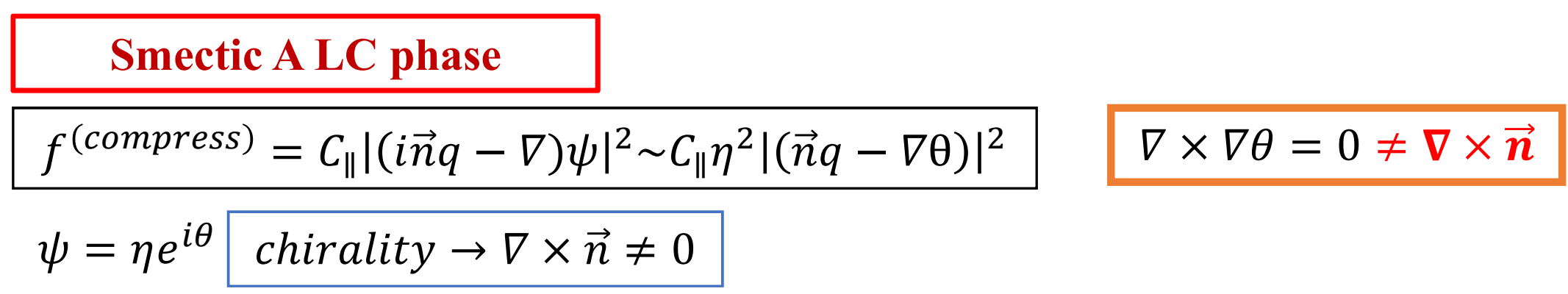

$$
\begin{array}{r}
\hline \text { Superconductor } \\
\left|f^{(\text {coupl })} \sim \frac{\hbar^{2}}{4 m} \eta^{2}\right| \nabla \theta-\left.\frac{2 e}{\hbar c} \vec{A}\right|^{2} \\
\nabla \times \nabla \theta=0 \neq \nabla \times \vec{A}=\vec{B}
\end{array}
$$




\section{Minimal model}

$$
\mathrm{f}=\kappa \operatorname{Tr} \underline{C}^{2}-\alpha \operatorname{Tr} \underline{Q}^{2}+\beta / 2\left(\operatorname{Tr} \underline{Q}^{2}\right)^{2}+\mathrm{k}_{i}|\nabla \underline{Q}|^{2}+\mathrm{k}_{e} \underline{Q} \cdot \underline{C}^{2}
$$

$$
\underline{Q} \rightarrow \underline{Q} / \lambda_{0} \quad \lambda_{0}=\sqrt{\alpha / \beta} \quad \underline{C} \rightarrow \underline{C} / R_{0}
$$

$$
\mathrm{f}=\frac{\kappa}{R_{0}^{2}} \operatorname{Tr} \underline{C}^{2}+\alpha \lambda_{0}^{2}\left(-\operatorname{Tr} \underline{Q}^{2}+\frac{1}{2}\left(\operatorname{Tr} \underline{Q}^{2}\right)^{2}+\left(\frac{\xi}{R_{0}}\right)^{2}\left(|\nabla \underline{Q}|^{2}+\frac{1}{\lambda_{0}} \frac{\mathrm{k}_{e}}{\mathrm{k}_{i}} \underline{Q} \cdot \underline{C}^{2}\right)\right)
$$

Extrinsic term dominates close to a continuous phase transition!

$$
\text { Sci. Rep. 9, } 19742 \text { (2019). }
$$


Without extrinsic term :
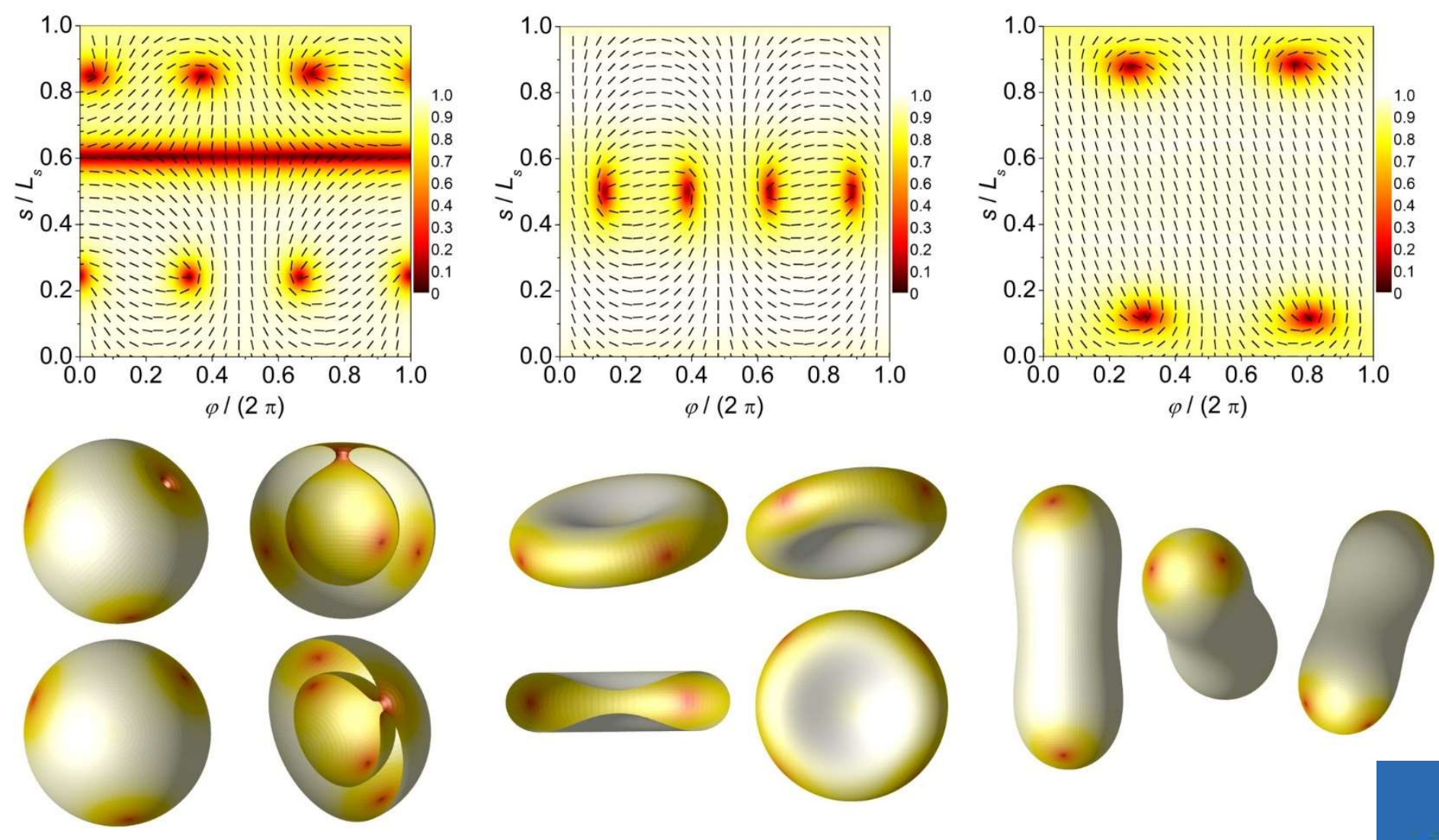

Crystals 2020 
With extrinsic term :
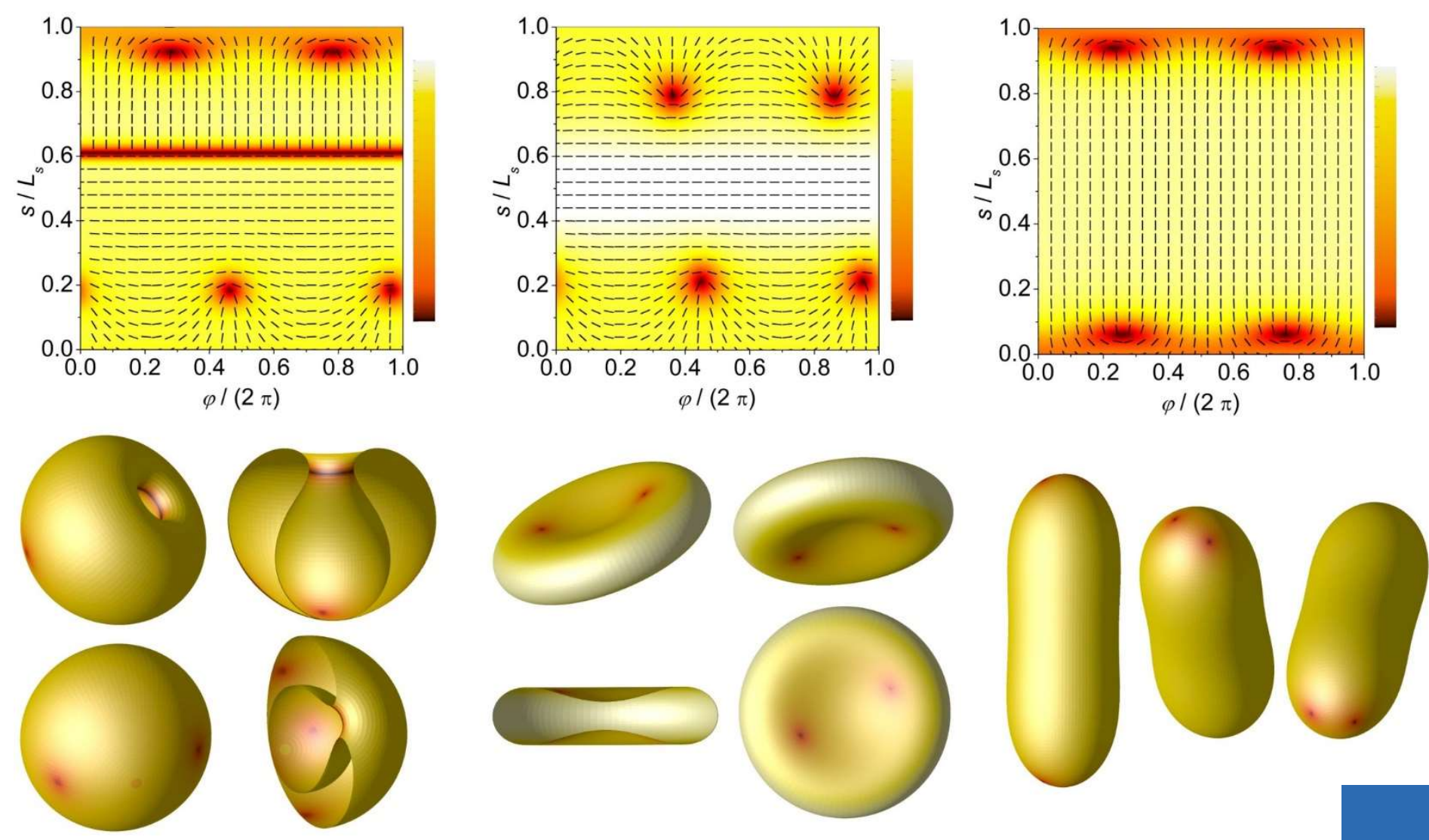

\section{Crystals 2020}




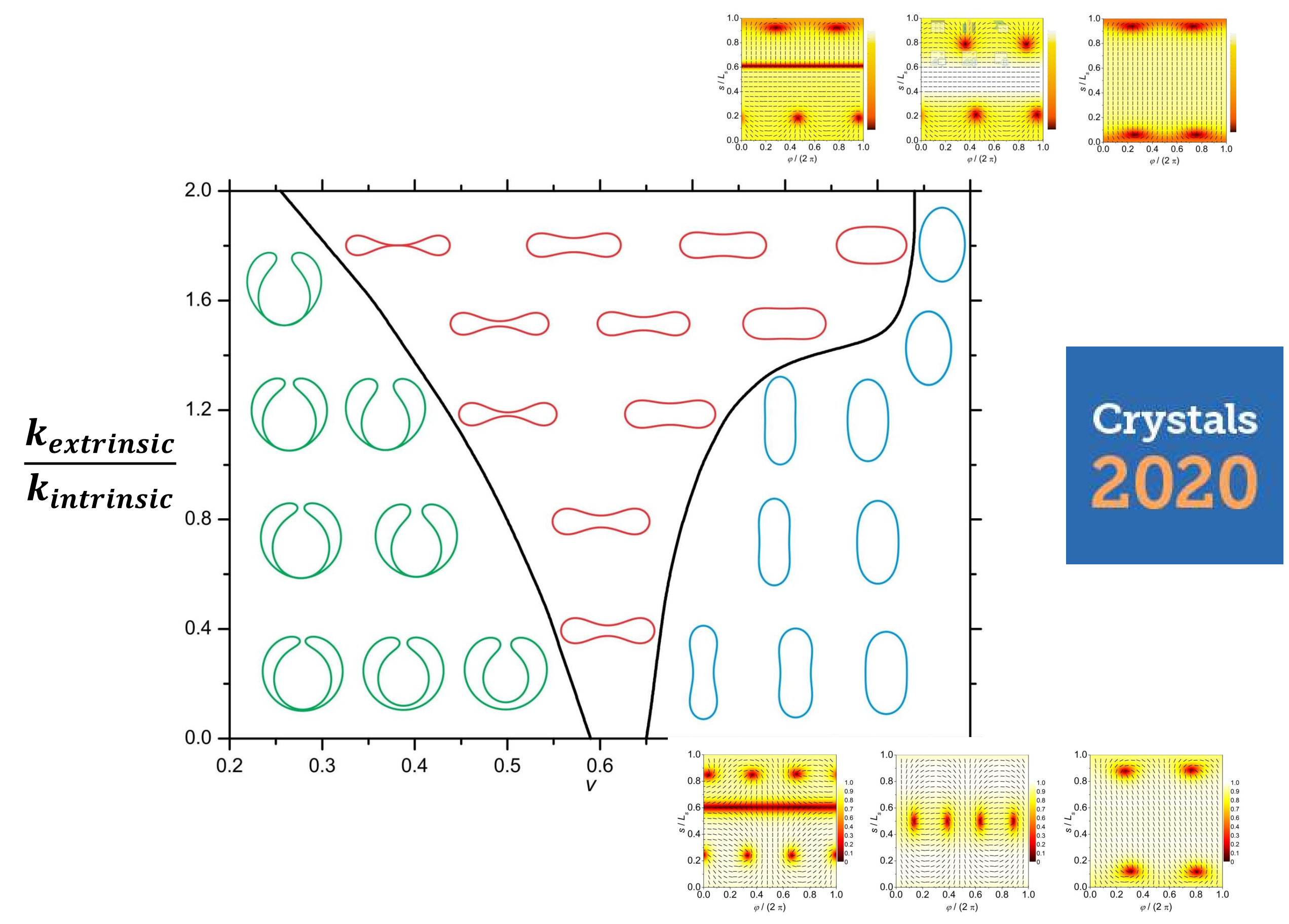




\section{Stabilisation of "chargeless" TDs}
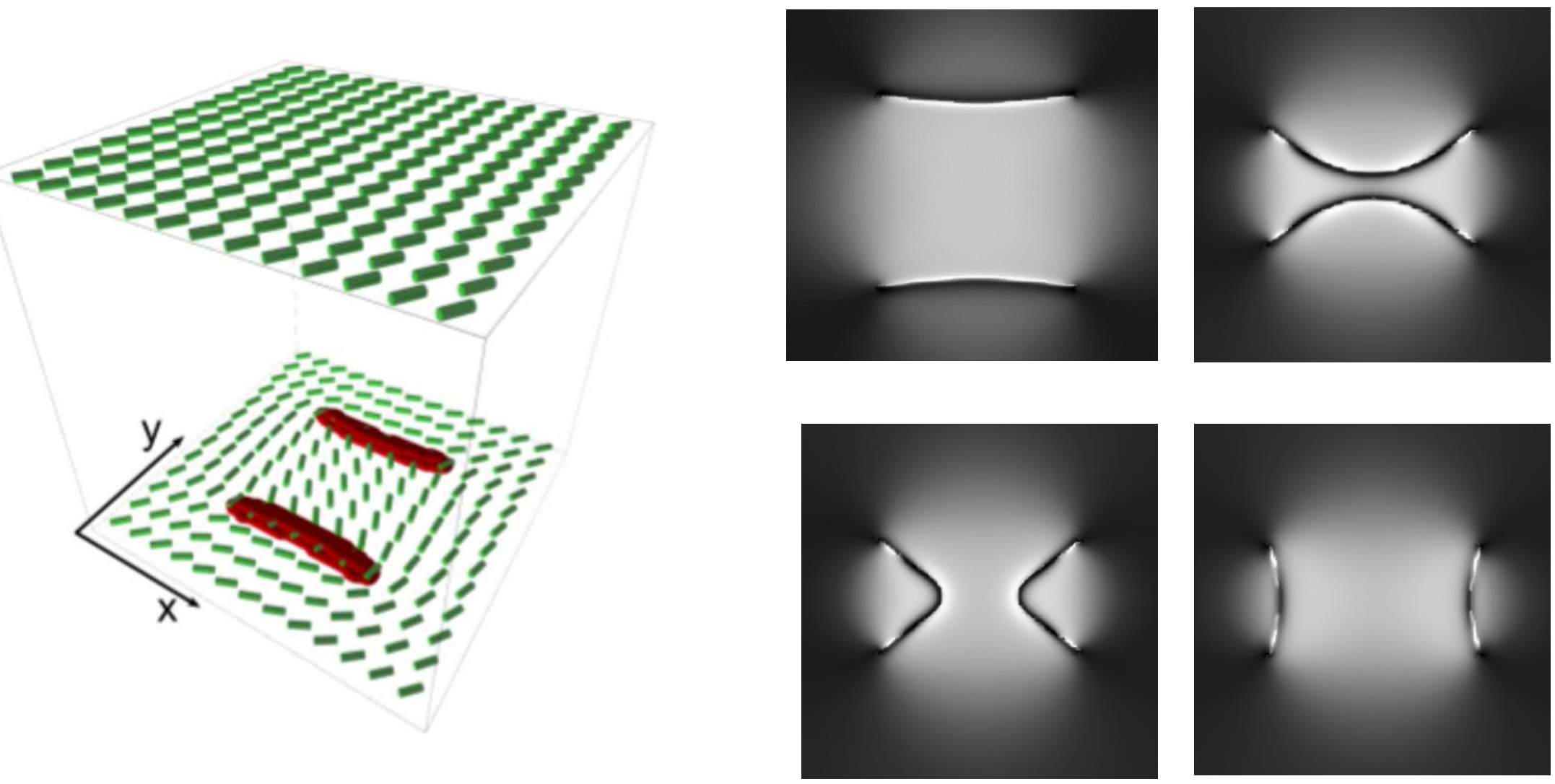

Crystals

2020 

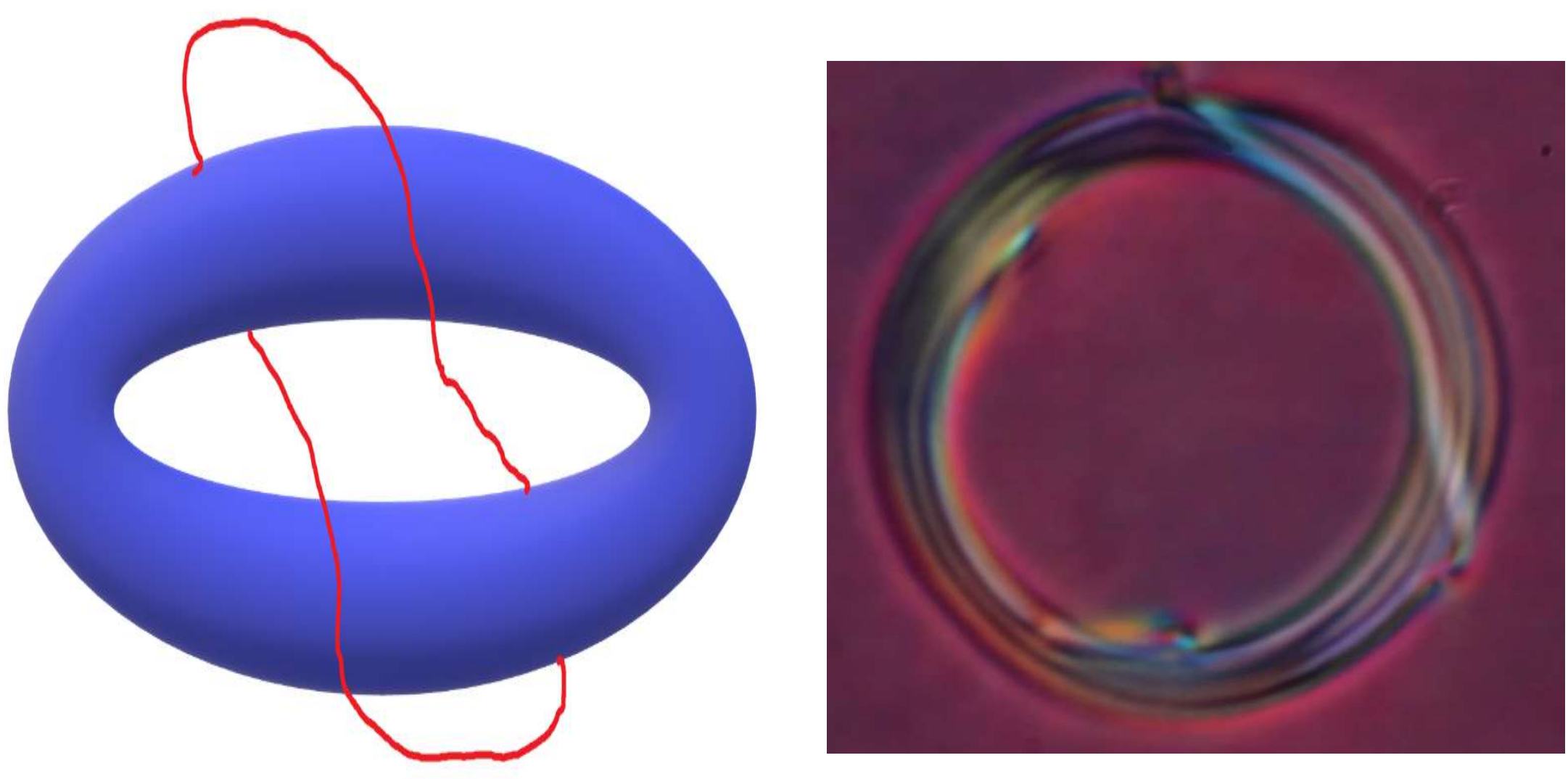

Crystals 2020 


\section{VACUUM STATE}

The Hidden Space

Any given solution to the equations of string theory represents a specific configuration of space and time. In particular, it specifies the arrangement of the small dimensions, along with their associated branes (green) and lines of force known as flux lines (orange). Our world has six extra dimensions, so every point of our familiar three-dimensional space hides an associated tiny six-dimensional space, or manifold-a six-dimensional analogue of the circle in the top illustration on page 81 . The physics that is observed in the three large dimensions depends on the size and the structure of the manifold: how many doughnutlike "handles" it has, the length and circumference of each handle, the number and locations of its branes, and the number of flux lines wrapped around each doughnut.

By Raphael Bousso and Joseph Polchinski
Brane

Flux line

Point in space

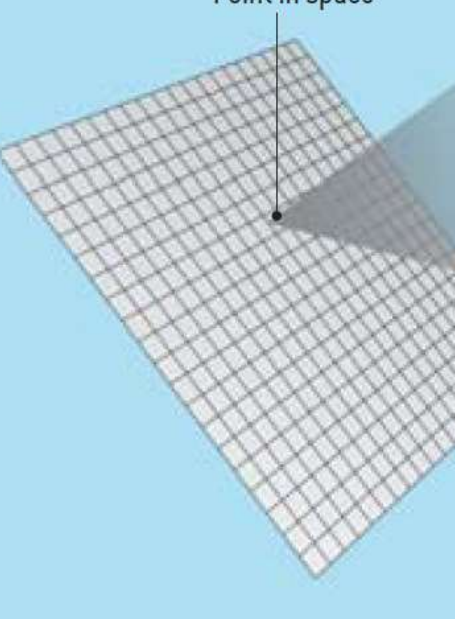

Space

\section{Manifold of extra dimensions}




\section{Stabilisation with nanoparticles}

\section{Strong enough chirality}
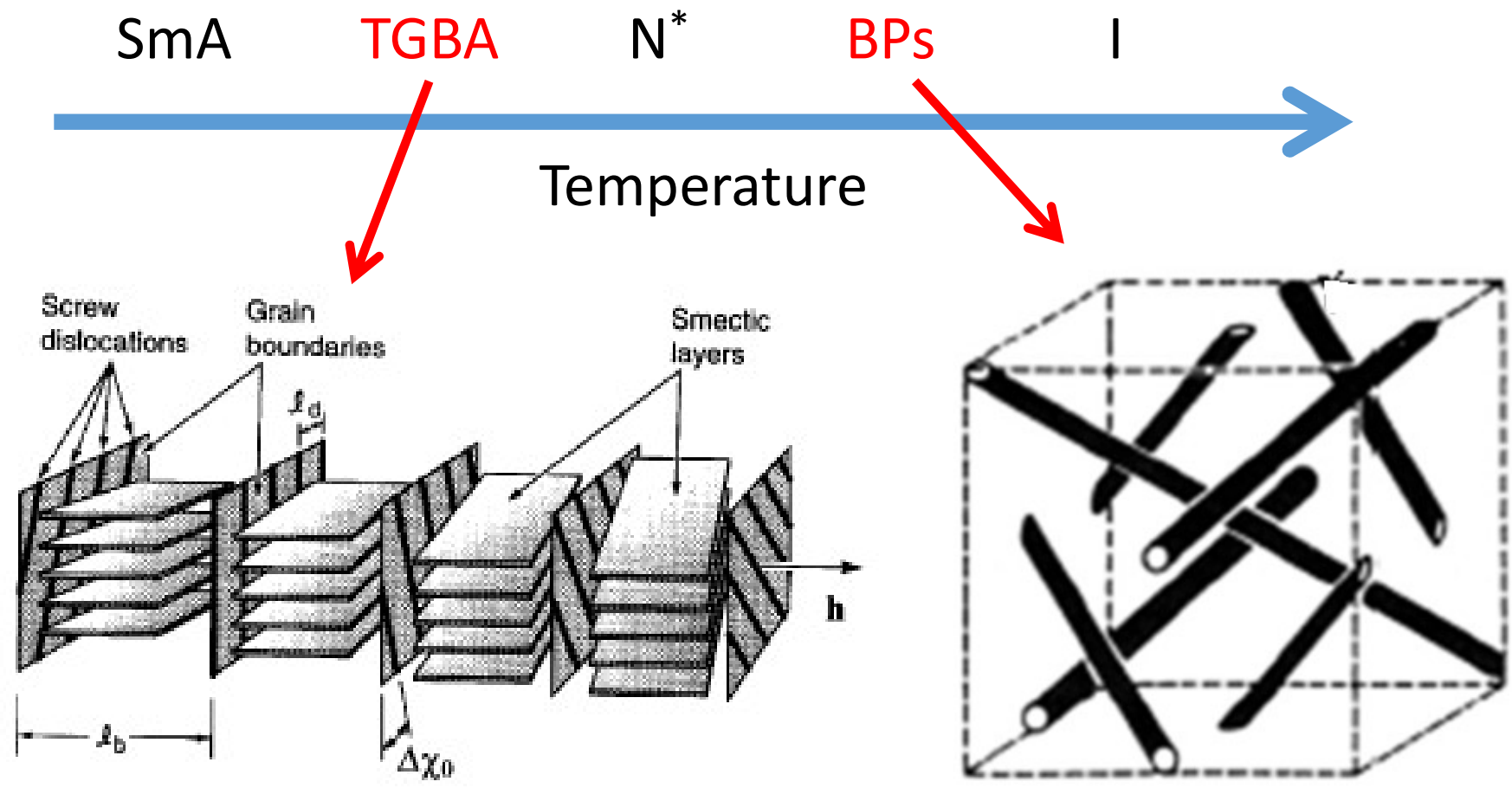

Renn\&Lubensky, Phys.Rev. A 38, 2132 (1988).

Wight\&Mermin, Rev.Mod.Phys. 61, 385 (1989). 
Chiral LCs : Blue Phases

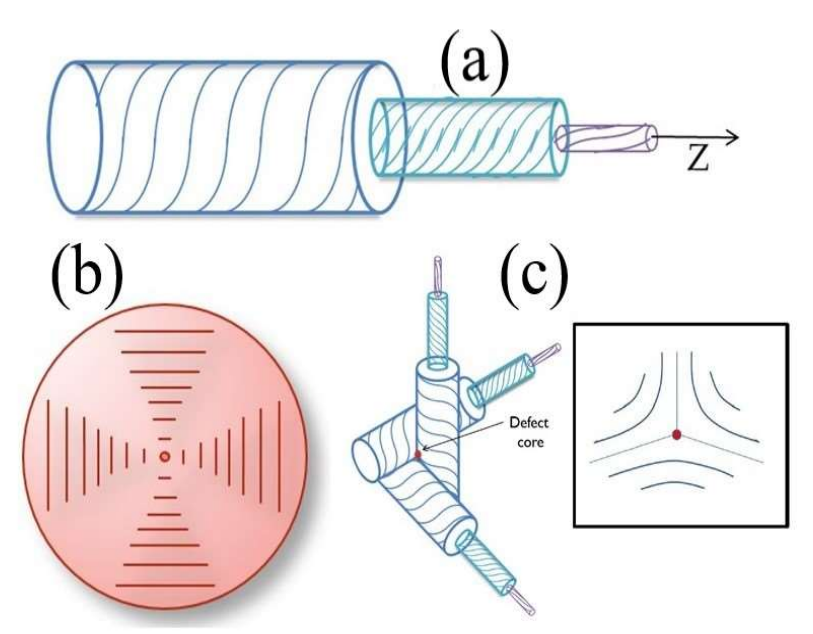

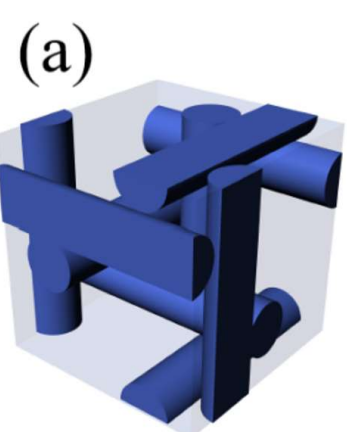

(b)
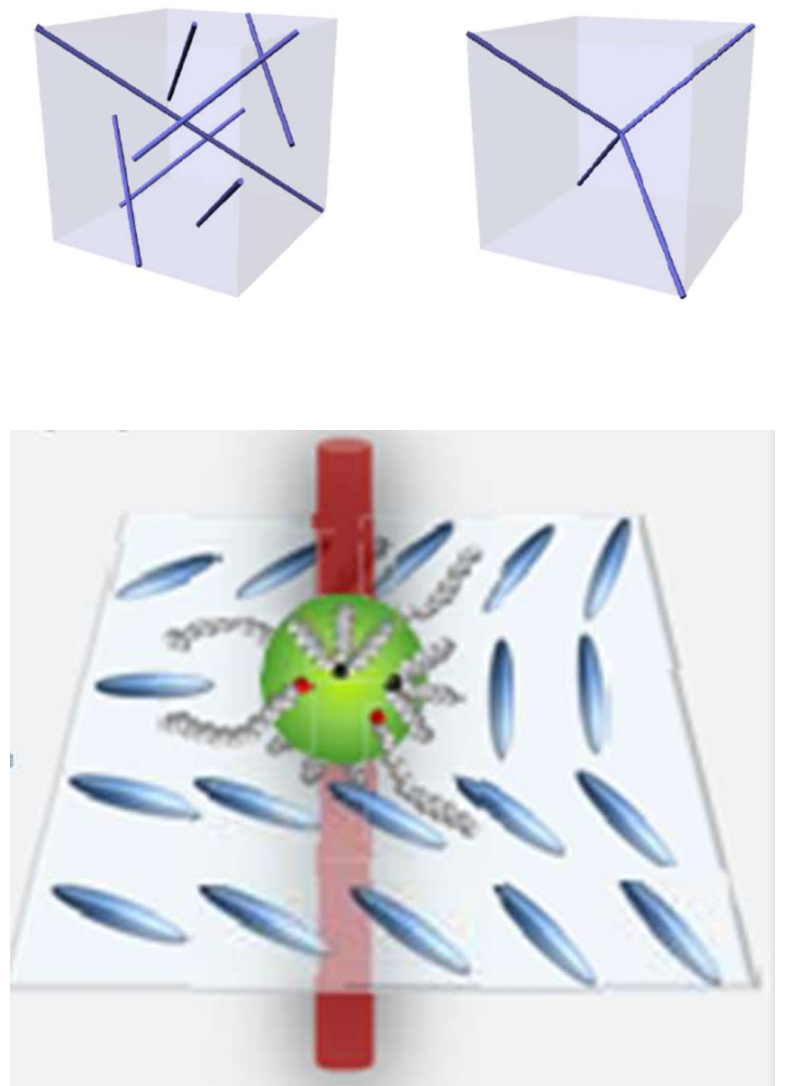

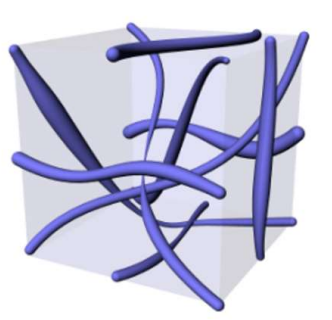

(c)

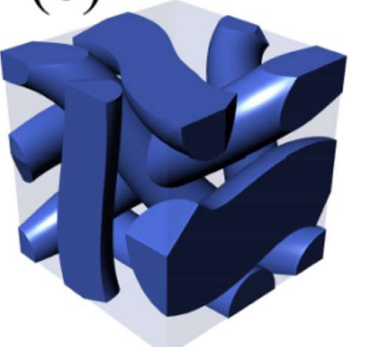

Crystals 2020 
Twist grain boundary phases
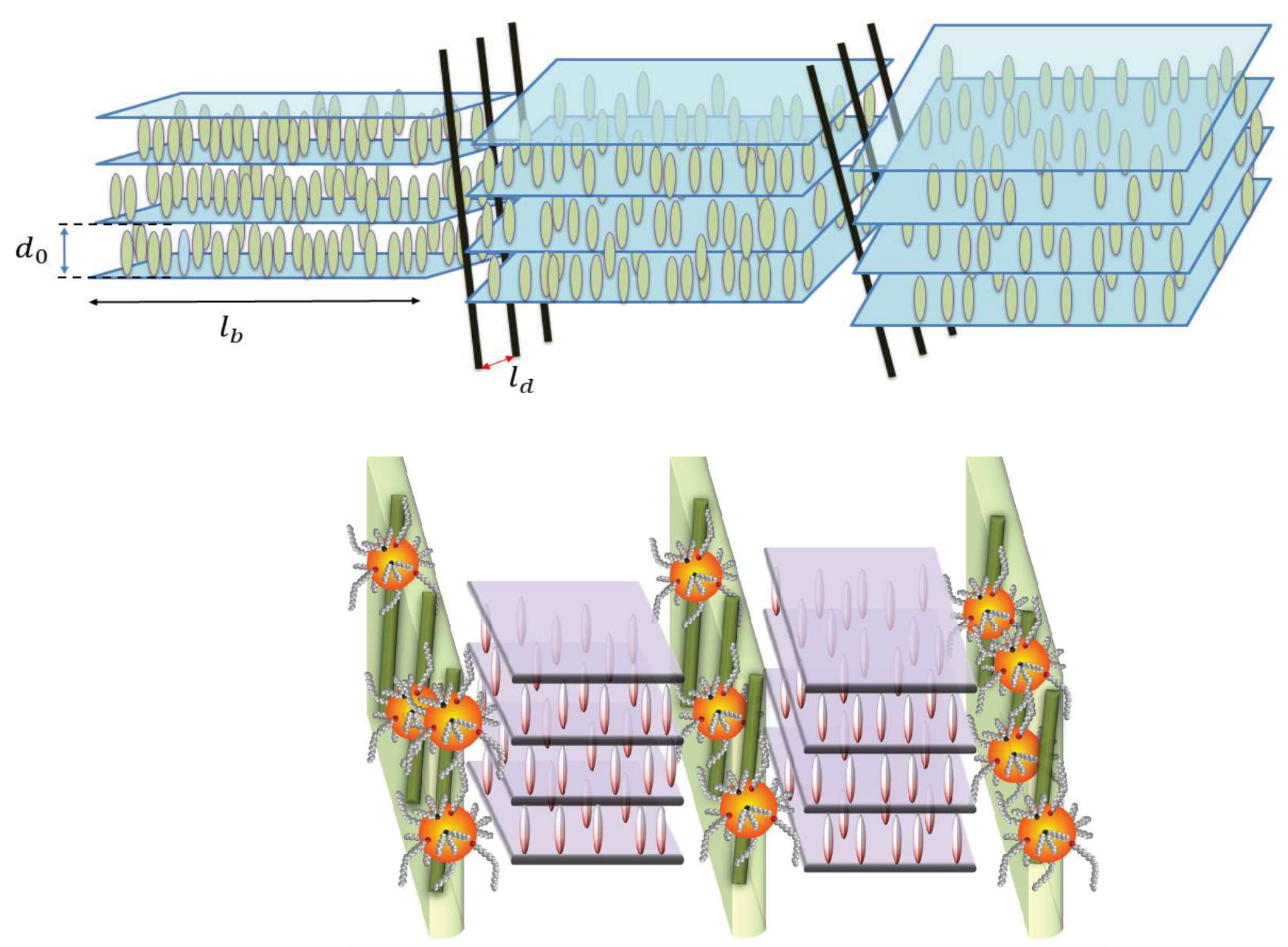

Crystals 2020 


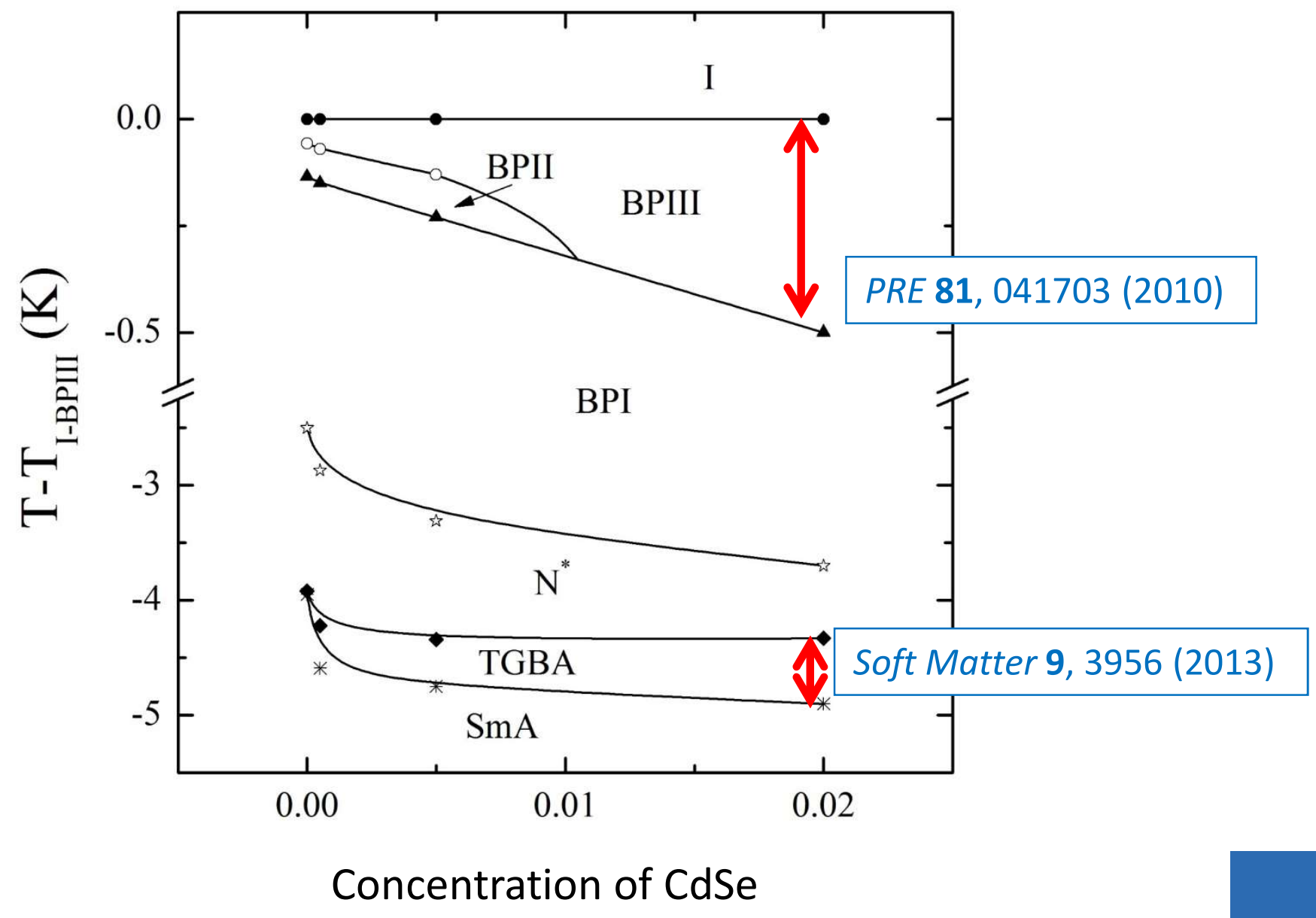

Crystals 2020 


\section{Conclusions}

- Stabilisation of defects: robust manipulations among different stable configurations of defects

- Potential applications: rewirable conductive wires, information storage, photonics, sensors...

- Fundamental science: fields as fundamental entities 